\title{
CONSISTENCY ESTIMATES FOR A DOUBLE-LAYER POTENTIAL AND APPLICATION TO THE NUMERICAL ANALYSIS OF THE BOUNDARY-ELEMENT APPROXIMATION OF ACOUSTIC SCATTERING BY A PENETRABLE OBJECT
}

\author{
A. BENDALI AND M. SOUILAH
}

\begin{abstract}
The consistency of the approximation of a double-layer potential when using a boundary-element method and approximating the curved boundary by a polygonal curve in two-dimensional problems or by a polyhedral surface in three-dimensional ones is investigated. The results are applied to the numerical analysis of the approximation of a model problem: the diffraction of a time-harmonic acoustic wave by a penetrable object.
\end{abstract}

\section{INTRODUCTION}

Double-layer potentials are widely used in the numerical computation of solutions to boundary value problems by means of boundary-element methods. Without being exhaustive, we quote: Laplace and Helmholtz equations (cf. Nédélec [24], Giroire [12,13]), scattering of an electromagnetic wave by a dielectric cylinder (cf. Chang and Harrington [4]), coupling interior and boundary elements (cf. Johnson and Nédélec [18], Sequeira [34]), etc.

In the case of curved boundary, for reasons of effective computation and digital handling of geometrical data, an approximate boundary must be included on which the discrete problem is posed. Although this treatment is quite common in practical computations, as far as we know, no result is available on the consistency or the asymptotic behavior of the error due to this approximation. By contrast, for the single-layer potential, the method initiated by Nédélec [25] gives complete answers in several directions (cf., e.g., Le Roux [22], Giroire [12, 13], Bendali [2, 3], Johnson and Nédélec [18], etc.). This seems to be due to the following feature. The parametrization of the exact boundary is done by an orthogonal projection of the approximate boundary on the former (cf. Nédelec [25]). The consistency error is then essentially obtained by estimating the difference between the potential kernel values for two points on the approximate boundary and for their respective projections on the exact boundary. This could be called "a local technique estimate". Since the singularity of the derivatives of the kernel of single-layer potentials is generally not integrable, this approach is not suited for double-layer potentials. Therefore, a global procedure is needed. The aim of the present work is precisely to deal with this problem. The basic

Received by the editor October 25, 1990 and, in revised form, June 15, 1992.

1991 Mathematics Subject Classification. Primary 65N38; Secondary 65R20, 78A45, 76M15. 
idea is to use a Green's formula in the domain bounded by the actual boundary and the approximate one. Then the consistency estimate essentially results in relatively long calculations involving interior elliptic estimates, the so-called inverse property in the finite element method, and exact computation of the potential created by linear distribution on elements of the approximate boundary, initiated by De Hoop (cf. Van Herk [36]).

Among all the boundary value problems whose formulation as boundary integral equation involves a double-layer potential, the results obtained are illustrated by carrying out the numerical analysis of the one which is related to the scattering of a time-harmonic acoustic wave by a penetrable object. Two reasons essentially motivate this choice. The first comes from the fact that the kernel arising in this problem is that of the Helmholtz equation. It is decomposed as a superposition of the kernel of the static problem, which is that of the Laplace equation, and a less singular kernel to which Nédélec's "local technique estimate" applies. Hence, this situation gives answers which can be used in other directions. Beside the fact that this problem is important in itself in the applications, as suggested by the numerous papers devoted to the subject (cf., e.g., Harrington [16], Rokhlin [31], Kleinman and Martin [19], etc.), the second reason is due to the formulation of the problem as a Fredholm system of integral equations of the first kind, free of hypersingular integrals, considered here. Although the formulation as a Fredholm system of integral equations of the second kind is the most commonly used and the easiest to analyze, it is presently well established that the formulation considered in this paper has generally better numerical stability properties (cf. Nédélec [26]).

The formulation as a Fredholm system of the first kind is obtained directly in the two-dimensional case when modeling the scattering of a time-harmonic electromagnetic wave by a dielectric cylinder (cf. Chang and Harrington [4], Clair [6], for example). This formulation appears without the removal of the hypersingular integrals in the paper of Costabel and Stephan [9]. Hypersingular integrals are numerically difficult to handle. The normal trace of a doublelayer potential given variationally by Hamdi [15] is the procedure used here to remove the hypersingularities of the integrals from the formulation in both two- and three-dimensional cases. It must be emphasized that the formulation given by Chang and Harrington, starting from Maxwell's equations in the two-dimensional case, directly provides the correct formulation without using Hamdi's formula. It must also be noted that direct numerical approximations of hypersingular integrals recently became available (cf. Schwab and Wendland [32], Krishnasamy et al. [21]).

The paper is organized as follows. In $\S 1$ the mathematical statement of the problem of scattering of a time-harmonic acoustic wave by a penetrable obstacle is given. Some known results on existence, uniqueness, and regularity of the solution are recalled. The rest of $\S 1$ is devoted to the reduction of the transmission problem to an integro-differential system stated on the boundary of the obstacle. The discretization of this system by a finite element method constitutes the boundary-element method used for the approximation of the solution of the scattering problem. If the consistency error for the approximation of the double-iayer potential is not taken into account, the numerical analysis of the problem may be performed by techniques which are by now standard (cf. Nédélec [24], Le Roux [22], etc.). Therefore, $\S 2$ of this article is devoted entirely 
to this consistency error, and only the final results of the numerical analysis are given.

\section{THE SCATTERING PROBLEM AND ITS APPROXIMATION}

1.1. The boundary value problem. The acoustic equations (cf., e.g., Germain [11] for general equations and boundary conditions, and Kress and Roach [20] for particular aspects considered here) show that the time-harmonic wave $v$ scattered by a penetrable obstacle is a solution of the following transmissiontype boundary value problem.

With the notation

$$
\left\{\begin{array}{l}
\Omega_{1} \text { a bounded } C^{\infty} \text { domain of } \mathbb{R}^{n} ; n=2,3, \\
\Omega_{2}:=\mathbb{R}^{n} \backslash \bar{\Omega}_{1} \text { the exterior domain, }
\end{array}\right.
$$

$$
\left\{\begin{array}{l}
\text { find } v \in C^{\infty}\left(\bar{\Omega}_{1}\right) \cap C^{\infty}\left(\bar{\Omega}_{2}\right) \text { such that } \\
\Delta v+k_{1}^{2} v=\left(k_{2}^{2}-k_{1}^{2}\right) u_{0} \text { in } \Omega_{1}, \\
\Delta v+k_{2}^{2} v=0 \text { in } \Omega_{2}, \\
{[v]=0 \text { on } \Gamma,} \\
{\left[\chi \partial_{\mathbf{n}} v\right]=-\left[\chi \partial_{\mathbf{n}} u_{0}\right] \text { on } \Gamma,} \\
\lim _{|x| \rightarrow+\infty}|x|^{(n-1) / 2}\left(\operatorname{grad} v \cdot x /|x|-i k_{2} v\right)=0 .
\end{array}\right.
$$

The last relation appearing in (1.2) is the Sommerfeld radiation condition describing the energy propagation from the obstacle towards infinity. In connection with this latter condition, the limit is always assumed to be uniform with respect to the angular variables $x /|x|$.

In the preceding equations (1.2),

$\Gamma$ denotes the common boundary to $\Omega_{1}$ and $\Omega_{2}$,

n the unit normal to $\Gamma$ outwardly directed to $\Omega_{1}$,

$\chi$ a piecewise constant function defined by

$$
\chi:= \begin{cases}1 / \rho_{1} & \text { in } \Omega_{1}, \\ 1 / \rho_{2} & \text { in } \Omega_{2},\end{cases}
$$

where $\rho_{m}>0, m=1,2$, is the density of fluid at rest occupying the domain $\Omega_{m}$

$u_{0}$ the (given) incident wave (generally a plane wave) satisfying the equation

$$
\Delta u_{0}+k_{2}^{2} u_{0}=0 \quad \text { in a neighborhood of } \bar{\Omega}_{1},
$$

$k_{m}, m=1,2$, the wave number

$$
k_{m}:=\omega / c_{m},
$$

where $c_{m}$ is the sound velocity in the fluid occupying the domains $\Omega_{m}$; the time variation $e^{-i \omega t}(\omega>0$ is the pulsation) is suppressed by linearity,

$[\cdot]$ is the jump across $\Gamma$ defined by

$$
[w]:=\left.w^{(1)}\right|_{\Gamma}-\left.w^{(2)}\right|_{\Gamma},
$$

where $\left.w^{(m)}\right|_{\Gamma}, m=1,2$, is the trace on $\Gamma$ of a function or distribution defined in $\Omega_{m}$, 
$\mathbf{a} \cdot \mathbf{b}=\sum_{i=1}^{n} a_{i} \bar{b}_{i}$ is the "scalar product" of two vectors respectively with $n$ real or complex components $a_{i}, b_{i}, i=1, \ldots, n$.

Other notation, as well as some function spaces like the Schwartz spaces $\mathscr{D}^{\prime}\left(\Omega_{1}\right), \mathscr{D}\left(\Omega_{1}\right)$, etc., or Sobolev spaces like $H^{1}\left(\Omega_{1}\right), H^{1 / 2}(\Gamma)$, etc., are standard in partial differential equation theory (cf., e.g., Lions and Magenes [23], Chazarain and Piriou [5], etc.) and are used without further comment.

It must be emphasized that, as a result of $(1.1), \Gamma$ is a closed, bounded $(n-1)$-dimensional $C^{\infty}$ manifold without boundary, embedded in $\mathbb{R}^{n}$. In fact, this amount of smoothness is not really necessary for the subsequent analysis. It seems to be sufficient to assume that $\Gamma$ is of class $C^{2}$. Since, however, this point is not essential, neither for the main result nor for the techniques which are developed for its proof, we choose not to further complicate the exposition by this discussion.

In order to give an existence-uniqueness theorem for the problem (1.2), and for the purpose of numerical analysis, a problem set in a more general framework is considered.

Given $f$ in $L^{2}\left(\Omega_{1}\right), g$ in $H^{1 / 2}(\Gamma)$, and $\zeta$ in $H^{-1 / 2}(\Gamma)$,

$$
\left\{\begin{array}{l}
\text { find } v \in H^{1}\left(\Omega_{1}\right) \cap H_{\mathrm{loc}}^{1}\left(\bar{\Omega}_{2}\right) \text { such that } \\
\Delta v+k_{1}^{2} v=-f \text { in } \mathscr{D}^{\prime}\left(\Omega_{1}\right), \\
\Delta v+k_{2}^{2} v=0 \text { in } \mathscr{D}^{\prime}\left(\Omega_{2}\right), \\
{[v]=g \text { in } H^{1 / 2}(\Gamma),} \\
{\left[\chi \partial_{\mathbf{n}} v\right]=\zeta \text { in } H^{-1 / 2}(\Gamma),} \\
\lim _{|x| \rightarrow+\infty}|x|^{(n-1) / 2}\left(\operatorname{grad} v \cdot x /|x|-i k_{2} v\right)=0 .
\end{array}\right.
$$

The behavior of $v$ at infinity is described by the radiation condition and not by its belonging to any Sobolev space (cf. Wilcox [37]). This explains the involvement of the Fréchet space

$$
H_{\text {loc }}^{1}\left(\bar{\Omega}_{2}\right):=\left\{v \in \mathscr{D}^{\prime}\left(\Omega_{2}\right) \mid \phi v \in H^{1}\left(\Omega_{2}\right), \forall \phi \in \mathscr{D}\left(\mathbb{R}^{n}\right)\right\}
$$

in the formulation. For any real number $s$, the space $H_{\text {loc }}^{s}\left(\bar{\Omega}_{2}\right)$ is similarly defined.

The following theorem can then be stated.

Theorem 1.1. The problem (1.7) has one and only one solution. Moreover, for all $s \geq-1$, if the data respectively satisfy $f \in H^{\max (s, 0)}\left(\Omega_{1}\right), g \in H^{s+3 / 2}(\Gamma)$, and $\zeta \in H^{s+1 / 2}(\Gamma)$, the following regularity holds:

$$
v \in H^{s+2}\left(\Omega_{1}\right) \cap H_{\mathrm{loc}}^{s+2}\left(\bar{\Omega}_{2}\right) .
$$

Proof. Uniqueness follows by Rellich's lemma [30] (cf., e.g., Kress and Roach [20]). Existence of a solution can be obtained either by standard arguments involving the limiting absorption principle like in Wilcox [37], or by the elegant method of Lax and Phillips (cf. Phillips [29]). The higher-order regularity, $s>-1$, holds by general results for elliptic systems due to Agmon, Douglis, and Nirenberg [1].

\subsection{Integral equations. Let}

$$
u:=v+u_{0}
$$


where $v$ is the scattered wave by the obstacle, solution of problem (1.2), and $u_{0}$ is the incident wave. The representation formula for the solution of the Helmholtz equation satisfying an outgoing Sommerfeld radiation condition in the case of an exterior domain (cf., e.g., Colton and Kress [7], Gunter [14]) gives

$$
u(x)= \begin{cases}\int_{\Gamma}\left(G_{1}(x, y) \lambda^{(1)}(y)-\partial_{\mathbf{n}} G_{1}(x, y) p^{(1)}(y)\right) d \Gamma(y), & x \in \Omega_{1}, \\ u_{0}(x)-\int_{\Gamma}\left(G_{2}(x, y) \lambda^{(2)}(y)-\partial_{\mathbf{n}} G_{2}(x, y) p^{(2)}(y)\right) d \Gamma(y), & x \in \Omega_{2} .\end{cases}
$$

Here, $G_{m}$ is the Green's kernel giving the outgoing solutions of the Helmholtz equation,

$$
G_{m}(x, y):=\frac{i}{4}\left(\frac{k_{m}}{2 \pi|x-y|}\right)^{(n-2) / 2} H_{(n-2) / 2}^{(1)}\left(k_{m}|x-y|\right), \quad x \neq y, \quad m=1,2,
$$

where $H_{t}^{(1)}$ is the Hankel function of order 1 and index $t$ (cf., e.g., Wilcox [37]), and

$$
\left\{\begin{array}{l}
p^{(m)}:=\left.\mathcal{u}^{(m)}\right|_{\Gamma}, \\
\lambda^{(m)}:=\left.\partial_{\mathbf{n}} u^{(m)}\right|_{\Gamma}, \quad m=1,2,
\end{array}\right.
$$

are the respective first and the second trace of $u$ taken from its values on $\Omega_{m}$, $m=1,2$. Unless mentioned explicitly otherwise, all derivatives occurring under the integral sign are implicitly assumed to be taken with respect to the variable $y$.

The transmission conditions then show that the determination of $u$ is reduced to that of

$$
\begin{aligned}
& \lambda:=\lambda^{(1)} / \rho_{1}=\lambda^{(2)} / \rho_{2}, \\
& p:=p^{(1)}=p^{(2)} .
\end{aligned}
$$

The formulae, giving the traces of single- and double-layer potentials, yield

$$
\left\{\begin{array}{l}
\rho_{1} V_{1} \lambda-N_{1} p=p / 2, \\
\rho_{2} V_{2} \lambda-N_{2} p=-p / 2+\left.u_{0}\right|_{\Gamma}, \\
K_{1} \lambda-\frac{1}{\rho_{1}} D_{1} p=\lambda / 2, \\
K_{2} \lambda-\frac{1}{\rho_{2}} D_{2} p=-\lambda / 2+\left.\frac{1}{\rho_{2}} \partial_{\mathbf{n}} u_{0}\right|_{\Gamma},
\end{array}\right.
$$

where the integral operators involved on $\Gamma$ are denoted by

$$
\left\{\begin{array}{l}
V_{m} \lambda(x):=\int_{\Gamma} G_{m}(x, y) \lambda(y) d \Gamma(y), \\
N_{m} p(x):=\int_{\Gamma} \partial_{\mathbf{n}_{y}} G_{m}(x, y) p(y) d \Gamma(y), \\
K_{m} \lambda(x):=\int_{\Gamma} \partial_{\mathbf{n}_{x}} G_{m}(x, y) \lambda(y) d \Gamma(y), \\
D_{m} p(x):=\partial_{\mathbf{n}_{x}} \int_{\Gamma} \partial_{\mathbf{n}_{y}} G_{m}(x, y) p(y) d \Gamma(y),
\end{array} \text { for } x \in \Gamma \text { and } m=1,2 .\right.
$$


It is worth noting that the integrals defining $V_{m}, N_{m}$, and $K_{m}$ exist as usual Lebesgue integrals, whereas the last operator $D_{m}$ is hypersingular. Among all the possible methods which can be used to give it a meaning, the easiest and fastest, in the present context, is that in the sense of distributions (cf. Costabel [8] for a more general setting).

The mapping properties of these operators are as follows:

$$
\left\{\begin{array}{l}
V_{m}: H^{-1 / 2+s}(\Gamma) \rightarrow H^{1 / 2+s}(\Gamma), \\
N_{m}: H^{-1 / 2+s}(\Gamma) \rightarrow H^{1 / 2+s}(\Gamma), \\
K_{m}: H^{-1 / 2+s}(\Gamma) \rightarrow H^{1 / 2+s}(\Gamma), \\
D_{m}: H^{1 / 2+s}(\Gamma) \rightarrow H^{-1 / 2+s}(\Gamma)
\end{array}\right.
$$

for $m=1,2$ and for all real $s$ if $\Gamma$ is $C^{\infty}$ (cf., e.g., Chazarain and Piriou [5] and Seeley [33]). Costabel [8] has shown that the first and the last relations of (1.17) remain true if $\Gamma$ is only Lipschitz for $s<1 / 2$.

A Fredholm system of the first kind can be obtained by adding the first to the second equation, and the third to the last one. Then, multiplying by test functions and integrating once more over $\Gamma$ leads to the following variational system:

Given $(f, \zeta):=\left(\left.u_{0}\right|_{\Gamma},-\left.\left(1 / \rho_{2}\right) \partial_{\mathbf{n}} u_{0}\right|_{\Gamma}\right) \in T:=H^{1 / 2}(\Gamma) \times H^{-1 / 2}(\Gamma)$,

$$
\left\{\begin{array}{cc}
\text { find }(\lambda, p) \in Z:=H^{-1 / 2}(\Gamma) \times H^{1 / 2}(\Gamma) \text { such that } \\
\langle V \lambda, \mu\rangle-\langle N p, \mu\rangle=\langle f, \mu\rangle, \quad \forall \mu \in H^{-1 / 2}(\Gamma), \\
-\overline{\langle q, K \lambda\rangle}+\overline{\langle q, D p\rangle}=\overline{\langle q, \zeta\rangle}, \quad \forall q \in H^{1 / 2}(\Gamma),
\end{array}\right.
$$

where

$$
\left\{\begin{array}{l}
\langle\cdot, \cdot\rangle \text { is the (anti) duality pairing between } H^{1 / 2}(\Gamma) \text { and } H^{-1 / 2}(\Gamma), \\
V:=\rho_{1} V_{1}+\rho_{2} V_{2} \\
N:=N_{1}+N_{2} \\
K:=K_{1}+K_{2} \\
D:=\frac{1}{\rho_{1}} D_{1}+\frac{1}{\rho_{2}} D_{2} .
\end{array}\right.
$$

This formulation was given by Costabel and Stephan [9]. It needs the computation of the hypersingular integrals which appear when the explicit expression of the operator $D$ is considered. These hypersingular integrals are well understood from a theoretical point of view, thanks to the Calderon-Zygmund theory of singular integrals (cf., e.g., Neri [28]) or to the theory of pseudodifferential operators (cf., e.g., Chazarain and Piriou [5], Seeley [33], etc.). It is only recently, as was pointed out to the authors by the referee, that suitable quadrature schemes for finite part integrals (cf. Schwab and Wendland [32]) or regularization techniques based on Taylor expansion were proposed to treat directly the hypersingular operator $D$.

Here we choose to use the following recipe introduced by Hamdi [15] (cf. also Nédélec [26]), which essentially is an integration by parts on the boundary and which was used with some success in practical computations (cf., e.g., Chang and Harrington [4], Clair [6], etc.):

$$
\overline{\langle q, D p\rangle}=-c(p, q)+d(p, q),
$$




$$
\begin{gathered}
c(p, q):=\int_{\Gamma} \int_{\Gamma}\left(\frac{1}{\rho_{1}} G_{1}+\frac{1}{\rho_{2}} G_{2}\right)(x, y) \operatorname{grad}_{\Gamma} p(y) \times \mathbf{n}(y) \\
\cdot \operatorname{grad}_{\Gamma} \overline{q(x)} \times \mathbf{n}(x) d \Gamma(y) d \Gamma(x), \\
d(p, q):=\int_{\Gamma} \int_{\Gamma}\left(\frac{k_{1}^{2}}{\rho_{1}} G_{1}+\frac{k_{2}^{2}}{\rho_{2}} G_{2}\right)(x, y) p(y) \mathbf{n}(y) \\
\cdot \overline{q(x)} \mathbf{n}(x) d \Gamma(y) d \Gamma(x),
\end{gathered}
$$

where the multiplication sign $\times$ denotes the exterior vector product $\mathbf{a} \times \mathbf{b}:=$ $a_{1} b_{2}-a_{2} b_{1}$ in two-dimensional problems and the usual vectorial product in three dimensions, and $\operatorname{grad}_{\Gamma}$ is the tangential gradient of a function defined on $\Gamma$. It is worth noting that, in the two-dimensional case, $\operatorname{grad}_{\Gamma} p(y) \times \mathbf{n}(y)=\partial_{\sigma} p(y)$, where $\sigma$ is the curvilinear abcissa which parametrizes $\Gamma$ in the counterclockwise direction.

The operator $K$ is the transpose of the operator $N$; i.e., it satisfies

$$
\overline{\langle q, K \lambda\rangle}=\overline{\langle\bar{N} q, \lambda\rangle}, \quad \forall q \in H^{1 / 2}(\Gamma),
$$

where the operator $\bar{N}$ is defined by

$$
\bar{N} q=\overline{N \bar{q}} .
$$

With the sesquilinear forms

$$
\left\{\begin{array}{l}
a(\lambda, \mu):=\langle V \lambda, \mu\rangle, \\
b(p, \mu):=\langle N p, \mu\rangle, \\
b^{*}(\lambda, q):=\overline{\langle\bar{N} q, \lambda\rangle},
\end{array}\right.
$$

the system (1.18) can be rewritten in the form

$$
\left\{\begin{array}{l}
\text { find }(\lambda, p) \in Z \text { such that } \\
a(\lambda, \mu)-b(p, \mu)=\langle f, \mu\rangle, \quad \forall \mu \in H^{-1 / 2}(\Gamma), \\
-b^{*}(\lambda, q)-c(p, q)+d(p, q)=\overline{\langle q, \zeta\rangle}, \quad \forall q \in H^{1 / 2}(\Gamma) .
\end{array}\right.
$$

It is well known by now that the operator associated with the system (1.18) is a strongly elliptic pseudodifferential operator on $\Gamma$ (cf. Costabel and Wendland [10]). Usual duality properties in Hilbert spaces enable one to identify the space $T$ with the (anti)dual space $Z^{\prime}$ of $Z$. Then, the theory of pseudodifferential operators (cf., e.g., Chazarain and Piriou [5], Seeley [33]) can be used to decompose this operator in the form $\Lambda+\Theta$, where $\Lambda$ is coercive on $Z$, and to establish that $\Lambda$, for all real $s$, is a continuous mapping

$$
\Lambda: Z_{s} \rightarrow T_{s}
$$

and $\Theta$ a linear compact map from $Z_{s}$ into $T_{s}$, where $Z_{s}:=H^{-1 / 2+s}(\Gamma) \times$ $H^{1 / 2+s}(\Gamma)$ and $T_{s}:=H^{1 / 2+s}(\Gamma) \times H^{-1 / 2+s}(\Gamma)$.

The uniqueness of the solution of the system (1.18) (cf., e.g., Costabel and Stephan [9]) and the Fredholm alternative then yield the following theorem.

Theorem 1.2. The operators $\Lambda$ and $\Lambda+\Theta$ are (algebraic and topological) isomorphisms from $Z_{s}$ onto $T_{s}$ for all real $s$.

Remark 1.3. In view of the coerciveness properties given by Nédélec and Planchard [27] in the three-dimensional case, and by Hsiao and Wendland [17] in 
the two-dimensional case, it is actually a simple exercise to explicitly write out the system (1.26), up to some compact operators, as two uncoupled coercive variational equations. This leads to a simple and direct treatment of the error coming from the approximation of the boundary (cf. Bendali [3], where this approach is detailed for a similar case).

1.3. The boundary element method. According to Nédélec's ideas [25], the approximation of the curved exact boundary $\Gamma$ seems to require a complicated approach. It is assumed that there exists a finite family $\mathscr{F}$ of local charts (i.e., local coordinate patches) $(\omega, \psi)$, forming an atlas of $\Gamma$, and that the boundary $\Gamma$ can be "triangulated" using this atlas. More precisely, it is supposed that $\mathscr{F}$ is a finite family of triples $(\omega, D, \psi)$, where $D$ is a bounded segment in the twodimensional case and a polygonal domain in the three-dimensional case having its closure $\bar{D}$ contained in $\omega$, which further fulfills the following conditions:

$$
\Gamma=\bigcup_{(\omega, D, \psi) \in \mathscr{F}} \psi(\bar{D}),
$$

and the $\psi(\bar{D})$ are nonoverlapping and such that no vertex of one $\psi_{1}\left(\overline{D_{1}}\right)$ lies on the (interior of the) curvilinear edge of another $\psi_{2}\left(\overline{D_{2}}\right)$.

For all $(\omega, D, \psi) \in \mathscr{F}$, consider a "triangulation" $\mathscr{T}_{h}(D)$ of the domain $D$, in the usual meaning of finite element triangulations, with elements being segments in the two-dimensional case and triangles in the three-dimensional case. This leads to a triangulation $\mathscr{T}_{h}{ }^{*}$, a disjoint union of the $\mathscr{T}_{h}(D)$, which is assumed to satisfy the following uniform regularity condition:

$$
\left\{\begin{array}{l}
h_{T}:=\operatorname{diam}(T) \leq h, \quad \forall T \in \mathscr{T}_{h}^{*} ; \\
h / \rho_{T} \leq c,
\end{array}\right.
$$

here, $\rho_{T}$ denotes the diameter of the largest ball which can be inscribed in $T$. As usual in finite element error estimates, $c$ indicates various constants, not necessarily the same in all instances, but always independent of $h$ and of the solutions of the continuous or the discrete problem.

Let $(\omega, D, \psi)$ be in $\mathscr{F}$ and $T$ in $\mathscr{T}_{h}(D)$, and let $\psi_{T}$ be the linear interpolant of $\psi$ on $T$ from the values of $\psi$ on the vertices of $T$,

$$
K:=\psi_{T}(T)
$$

and $\Gamma_{h}$ the approximate boundary defined by

$$
\Gamma_{h}:=\bigcup_{\mathscr{F}} \bigcup_{T \in \mathscr{F}_{h}(D)} \psi_{T}(T)
$$

We denote by $\mathscr{T}_{h}$ the collection of all such elements $K$.

Following Nédélec [25], assume once again that the different triangulations are compatible in the following sense. With $\Gamma_{h}$ a closed polygonal curve in the two-dimensional case, and a polyhedral surface in the three-dimensional case, assume the above elements $K$ satisfy an overlapping condition similar to the one mentioned before. Observe that this construction forces the approximate boundary $\Gamma_{h}$ to have its vertices on the exact boundary $\Gamma$.

For all $K$ in $\mathscr{T}_{h}$, we introduce an orthonormal frame of the linear affine manifold supporting $K$ and let $\xi \in \mathbb{R}^{n-1}$ be the respective coordinates. When restricted to each $K$ in $\mathscr{T}_{h}$, the following definitions have an obvious meaning. 
A function $p$ defined on $K$ is said to belong to $\mathbb{P}_{m}$, the space of all complexvalued polynomials of degree less than or equal to $m$, if $p$, as function of the variable $\xi$, is in $\mathbb{P}_{m}$. By $\operatorname{grad}_{K} p$ we denote the tangent vector field to $K$ obtained in the usual way by differentiating with respect to the variable $\xi$. In this context, $\operatorname{grad}_{\Gamma_{h}} p$ will be the vector field tangent to $\Gamma_{h}$, defined almost everywhere by $\left.\operatorname{grad}_{\Gamma_{h}} p\right|_{K}:=\operatorname{grad}_{K} p$ for all $K$ in $\mathscr{T}_{h}^{*}$.

Let

$$
\begin{gathered}
M_{h}:=\left\{\lambda \in L^{2}\left(\Gamma_{h}\right)\left|\lambda_{K}:=\lambda\right|_{K} \in \mathbb{P}_{0}, \quad \forall K \in \mathscr{T}_{h}\right\}, \\
V_{h}:=\left\{q \in C^{0}\left(\Gamma_{h}\right)\left|q_{K}:=q\right|_{K} \in \mathbb{P}_{1}, \quad \forall K \in \mathscr{T}_{h}\right\} .
\end{gathered}
$$

The compatibility conditions made on the triangulation $\mathscr{T}_{h}{ }^{*}$ insure that the spaces $M_{h}$ and $V_{h}$ have dimensions given by the number of elements of $\mathscr{T}_{h}$ and the number of vertices of $\Gamma_{h}$, respectively.

We can then consider the approximate sesquilinear forms

$$
\begin{aligned}
a_{h}\left(\lambda_{h}, \mu_{h}\right):=\int_{\Gamma_{h}} \int_{\Gamma_{h}}\left(\rho_{1} G_{1}\left(x_{h}, y_{h}\right)+\rho_{2} G_{2}\left(x_{h}, y_{h}\right)\right) \\
\cdot \lambda_{h}\left(y_{h}\right) \overline{\mu_{h}\left(x_{h}\right)} d \Gamma_{h}\left(y_{h}\right) d \Gamma_{h}\left(x_{h}\right),
\end{aligned}
$$

for $\lambda_{h}$ and $\mu_{h}$ in $M_{h}$, and

$$
\begin{aligned}
b_{h}\left(p_{h}, \mu_{h}\right):=\int_{\Gamma_{h}} \int_{\Gamma_{h}}\left(\partial_{\mathbf{n}_{h}} G_{1}\left(x_{h}, y_{h}\right)+\partial_{\mathbf{n}_{h}} G_{2}\left(x_{h}, y_{h}\right)\right) \\
\cdot p_{h}\left(y_{h}\right) \overline{\mu_{h}\left(x_{h}\right)} d \Gamma_{h}\left(y_{h}\right) d \Gamma_{h}\left(x_{h}\right),
\end{aligned}
$$

for $p_{h}$ in $V_{h}$ and $\mu_{h}$ in $M_{h}$, and similar expressions for $b_{h}^{*}, c_{h}$, and $d_{h}$. The approximate (anti)linear forms are introduced as follows:

$$
\left\{\begin{array}{l}
\left\langle f_{h}, \mu_{h}\right\rangle_{h}:=\int_{\Gamma_{h}} u_{0}\left(x_{h}\right) \overline{\mu_{h}\left(x_{h}\right)} d \Gamma_{h}\left(x_{h}\right) \quad \text { for } \mu_{h} \text { in } M_{h}, \\
\overline{\left\langle q_{h}, \zeta_{h}\right\rangle_{h}}:=-\frac{1}{\rho_{2}} \int_{\Gamma_{h}} \partial_{\mathbf{n}_{h}} u_{0}\left(x_{h}\right) \overline{q_{h}\left(x_{h}\right)} d \Gamma_{h}\left(x_{h}\right) \text { for } q_{h} \text { in } V_{h} .
\end{array}\right.
$$

In this paper, for the sake of clarity, points lying on the approximate boundary and geometrical quantities related to $\Gamma_{h}$ are denoted with the help of an index $h$ to distinguish them from the corresponding objects on the exact boundary $\Gamma$. Moreover, there is a precise relation between a point denoted by $x$ on $\Gamma$ and the point $x_{h}$ on $\Gamma_{h}$, which is given in what follows.

We endow $M_{h}$ and $V_{h}$ with different norms. To this end, we begin by defining transport operators which carry each function defined on $\Gamma_{h}$ to a function defined on $\Gamma$. Following Nédélec [25], we consider the $\varepsilon$-tubular neighborhood of $\Gamma$,

$$
\mathscr{V}_{\varepsilon}:=\left\{x \in \mathbb{R}^{n} \mid \exists y \in \Gamma \text { such that }|x-y|<\varepsilon\right\} .
$$

If $\varepsilon$ is taken sufficiently small, the mapping $\Gamma \times(-\varepsilon, \varepsilon) \rightarrow \mathscr{V}_{\varepsilon}$ defined by $(m, t) \mapsto m+t \mathbf{n}(m)$ is a $C^{\infty}$ diffeomorphism (of manifolds), and the projection $\mathscr{P}: \mathscr{V}_{\varepsilon} \rightarrow \Gamma$ given by $\mathscr{P}(m+t \mathbf{n}(m))=m$ an infinitely differentiable map (in the meaning of differentiable maps of manifolds). Hereafter, assuming that such an $\varepsilon$ is fixed, there is an $h^{*}$ such that every $h$ less than $h^{*}$ gives rise to an approximate boundary $\Gamma_{h}$ contained in $\mathscr{V}_{\varepsilon}$. Then $\left.\mathscr{P}\right|_{\Gamma_{h}}$ defines a 
homeomorphism from $\Gamma_{h}$ onto $\Gamma$. In the sequel, it will always be assumed that every approximate boundary considered fulfills the later condition.

Hence, for all $K$ in $\mathscr{T}_{h}$,

$$
\widetilde{K}:=\mathscr{P} K .
$$

A parametrization of a neighborhood of $\widetilde{K}$ can then be obtained by

$$
x=\mathscr{P} x_{h}(\xi),
$$

where $x_{h}(\xi)$ is a point (of the linear manifold supporting $K$ ) in a neighborhood of $K$ with $\xi$ as coordinate.

The Lebesgue measure on $\Gamma$ may be written as

$$
d \Gamma(x):=\sqrt{g_{K}(\xi)} d \xi, \quad x=\mathscr{P} x_{h}(\xi) .
$$

As a result, the transport operators are defined by

$$
\left\{\begin{array}{l}
r_{h}: M_{h} \rightarrow L^{2}(\Gamma), \\
s_{h}: V_{h} \rightarrow C^{0}(\Gamma), \\
\left.\left(r_{h} \lambda_{h}\right)\right|_{\tilde{K}}(x):=\frac{1}{g_{K}(\xi)} \lambda_{K}, \quad x=\mathscr{P} x_{h}(\xi), \quad x_{h} \in K, \quad \forall K \in \mathscr{T}_{h}, \\
\left.\left(s_{h} q_{h}\right)\right|_{\widetilde{K}}(x):=q_{K}(\xi), \quad x=\mathscr{P} x_{h}(\xi), \quad x_{h} \in K, \forall K \in \mathscr{T}_{h} .
\end{array}\right.
$$

Remark 1.4. The multiplicative term $1 / \sqrt{g_{K}}$ is introduced to simplify some expressions occurring later. All norms written with or without this factor are uniformly equivalent relative to $h$.

Clearly, the operators $r_{h}$ and $s_{h}$ are one-to-one and can be used to identify respectively $V_{h}$ with a subspace of $C^{0}(\Gamma)$ and $M_{h}$ with a subspace of $L^{2}(\Gamma)$. Consequently, these spaces can be endowed with various norms

$$
\left\|\lambda_{h}\right\|_{s}:=\left\|r_{h} \lambda_{h}\right\|_{s, \Gamma}, \quad \lambda_{h} \in M_{h},
$$

for all $s \leq 0$, and

$$
\left\|p_{h}\right\|_{s}:=\left\|s_{h} p_{h}\right\|_{s, \Gamma}, \quad \lambda_{h} \in V_{h}
$$

for all $s \leq 1$.

Here, $\|\cdot\|_{s, \Gamma}$ is a norm of the Sobolev space $H^{s}(\Gamma)$ related to the previous atlas $\{(\omega, \psi) \mid(\omega, D, \psi) \in \mathscr{F}\}$.

All the following estimates are obtained by standard techniques (cf. Nédélec and Planchard [27], Nédélec [24], Le Roux [22], etc.).

Lemma 1.5 (Inverse inequalities). Under the assumption of uniform regularity of the triangulation $\mathscr{T}_{h}{ }^{*}$, we have

$$
\begin{gathered}
\left\|\lambda_{h}\right\|_{0} \leq c h^{-1 / 2}\left\|\lambda_{h}\right\|_{-1 / 2}, \quad \forall \lambda_{h} \in M_{h}, \\
\left\|p_{h}\right\|_{1} \leq c h^{-1 / 2}\left\|p_{h}\right\|_{1 / 2}, \quad \forall p_{h} \in V_{h}, \\
\left(\sum_{K \in \mathscr{F}_{h}} \operatorname{meas}(K)\left|\operatorname{grad}_{K} p_{K}\right|^{2}\right)^{1 / 2} \leq c h^{-1 / 2}\left\|p_{h}\right\|_{1 / 2}, \quad \forall p_{h} \in V_{h} .
\end{gathered}
$$

The consistency error of the approximation of the sesquilinear forms $a(\cdot, \cdot)$, $c(\cdot, \cdot)$ and $d(\cdot, \cdot)$ and of that of the (anti)linear forms $f$ and $\zeta$ are recalled in the following theorem. 
Theorem 1.6. The following consistency error estimates hold:

$$
\left\{\begin{array}{l}
\left|a\left(r_{h} \lambda_{h}, r_{h} \mu_{h}\right)-a_{h}\left(\lambda_{h}, \mu_{h}\right)\right| \leq c h\left\|\lambda_{h}\right\|_{-1 / 2}\left\|\mu_{h}\right\|_{-1 / 2}, \\
\left|c\left(s_{h} p_{h}, s_{h} q_{h}\right)-c_{h}\left(p_{h}, q_{h}\right)\right| \leq c h\left\|p_{h}\right\|_{1 / 2}\left\|q_{h}\right\|_{1 / 2}, \\
\left|d\left(s_{h} p_{h}, s_{h} q_{h}\right)-d_{h}\left(p_{h}, q_{h}\right)\right| \leq c h\left\|p_{h}\right\|_{1 / 2}\left\|q_{h}\right\|_{1 / 2}, \\
\left\langle f, \mu_{h}\right\rangle-\left\langle f_{h}, \mu_{h}\right\rangle_{h}\left|\leq c h^{3 / 2} \max _{\alpha=1} \sup _{x \in \mathscr{V}_{\varepsilon}}\right| \partial^{\alpha} u_{0}(x) \mid\left\|\mu_{h}\right\|_{-1 / 2}, \\
\left|\left\langle s_{h} q_{h}, \zeta\right\rangle-\left\langle q_{h}, \zeta_{h}\right\rangle_{h}\right| \leq c h \max _{|\alpha| \leq 2} \sup _{x \in \mathscr{V}_{\varepsilon}}\left|\partial^{\alpha} u_{0}(x)\right|\left\|q_{h}\right\|_{1 / 2},
\end{array}\right.
$$

for all $\lambda_{h}, \mu_{h}$ in $M_{h}$ and all $p_{h}, q_{h}$ in $V_{h}$.

In the above theorem, the incident wave $u_{0}$ is assumed to be defined and regular (at least $C^{2}$ ) in a tubular neighborhood $\mathscr{V}_{\varepsilon^{\prime}}$ with $\varepsilon^{\prime}>\varepsilon$.

Remark 1.7. It must be noticed that Le Roux's and Nédélec's results give the estimates

$$
\left|a\left(r_{h} \lambda_{h}, r_{h} \mu_{h}\right)-a_{h}\left(\lambda_{h}, \mu_{h}\right)\right| \leq c h^{2}\left\|\lambda_{h}\right\|_{0}\left\|\mu_{h}\right\|_{0},
$$

and only

$$
\left|d\left(s_{h} p_{h}, r_{h} q_{h}\right)-d_{h}\left(p_{h}, q_{h}\right)\right| \leq c h\left\|p_{h}\right\|_{0}\left\|q_{h}\right\|_{0},
$$

because of the loss of one convergence order when approximating the normal $\mathbf{n}$ to $\Gamma$ by the normal $\mathbf{n}_{h}$ to $\Gamma_{h}$. However, the use of the inverse inequality (1.45), to come back to the norm $\|\cdot\|_{-1 / 2}$, results in a loss of one convergence order in $h$ and makes the behavior of the consistency error of the two sesquilinear forms similar.

\section{CONSISTENCY ERROR ESTIMATE FOR A DOUBLE-LAYER POTENTIAL}

This section is almost entirely devoted to establishing the behavior of the consistency error for the approximation of the sesquilinear forms related to the double-layer potential, when the actual boundary is replaced by the approximate one. As it is pointed out in the introduction, this estimate cannot be obtained by the usual "local techniques". In order to make the discussion more straightforward, we decompose the kernel yielding the form $b(\cdot, \cdot)$ as a superposition of a singular part, which can be viewed as the kernel of the static case, and a less singular part, which describes the propagation properties of the solution. Obviously, the estimates related to the transposed form $b^{*}$ are similar. First, in $\S 2.1$, we state our main result and give one of its consequences, the numerical analysis of the scattering problem considered in the previous part. The next subsection is devoted to establishing the consistency estimate for the weakly singular part of the double-layer potential. The proof of the full consistency estimate is done in the last subsection and consists of several steps, each embodied by a lemma.

2.1. The main result and its consequence. The main result of this paper is as follows.

Theorem 2.1. There exists $h^{*}>0$ and a constant $c$ not depending on $0<h \leq$ $h^{*}$ such that

$$
\begin{gathered}
\left|b\left(s_{h} p_{h}, r_{h} \mu_{h}\right)-b_{h}\left(p_{h}, \mu_{h}\right)\right| \leq c h^{1 / 2}\left\|\mu_{h}\right\|_{-1 / 2}\left\|p_{h}\right\|_{1 / 2}, \\
\left|b^{*}\left(s_{h} p_{h}, r_{h} \mu_{h}\right)-b_{h}^{*}\left(p_{h}, \mu_{h}\right)\right| \leq c h^{1 / 2}\left\|\mu_{h}\right\|_{-1 / 2}\left\|p_{h}\right\|_{1 / 2},
\end{gathered}
$$

for all $p_{h}$ in $V_{h}$ and all $\mu_{h}$ in $M_{h}$. 
The discrete problem, for which the solution may be effectively computed, is stated as follows:

$$
\left\{\begin{array}{l}
\text { find }\left(\lambda_{h}, p_{h}\right) \in Z_{h}:=M_{h} \times V_{h} \text { such that } \\
a_{h}\left(\lambda_{h}, q_{h}\right)-b_{h}\left(p_{h}, \mu_{h}\right)=\left\langle f_{h}, \mu_{h}\right\rangle_{h}, \quad \forall \mu_{h} \in M_{h}, \\
-b_{h}^{*}\left(\lambda_{h}, q_{h}\right)-c_{h}\left(p_{h}, q_{h}\right)+d\left(p_{h}, q_{h}\right)={\overline{\left\langle q_{h}, \zeta_{h}\right\rangle_{h}}}, \quad \forall q_{h} \in V_{h},
\end{array}\right.
$$

where $\left\langle f_{h}, \mu_{h}\right\rangle_{h}$ and $\left\langle q_{h}, \zeta_{h}\right\rangle_{h}$ are suitable approximations of the linear maps occurring in the continuous problem defined through the data of $f$ in $H^{1 / 2}(\Gamma)$ and $\zeta$ in $H^{-1 / 2}(\Gamma)$.

Hence, using general results on the numerical analysis of nonconforming approximations of variational problems by a finite element method (cf., e.g., Strang and Fix [35]), Theorem 2.1 provides the last estimate needed to prove:

Theorem 2.2. There exists $h^{*}>0$ and a constant $c$ not depending on $0<h<$ $h^{*}$ such that for all $\left(f_{h}, \zeta_{h}\right)$ in $M_{h}^{\prime} \times V_{h}^{\prime}$, the discrete problem (2.3) has one and only one solution $\left(\lambda_{h}, p_{h}\right)$ that satisfies

$$
\begin{aligned}
\| r_{h} \lambda_{h} & -\lambda\left\|_{-1 / 2, \Gamma}+\right\| s_{h} p_{h}-p \|_{1 / 2, \Gamma} \\
\leq & \operatorname{ch}^{1 / 2}\left(\|f\|_{1 / 2, \Gamma}+\|\zeta\|_{-1 / 2, \Gamma}\right)+\sup _{\mu_{h} \in M_{h}} \frac{\left|\left\langle f_{h}, \mu_{h}\right\rangle_{h}-\left\langle f, r_{h} \mu_{h}\right\rangle\right|}{\left\|\mu_{h}\right\|_{-1 / 2}} \\
& \quad+\sup _{q_{h} \in V_{h}} \frac{\left|\left\langle q_{h}, \zeta\right\rangle_{h}-\left\langle s_{h} q_{h}, \zeta\right\rangle\right|}{\left\|q_{h}\right\|_{1 / 2}} .
\end{aligned}
$$

In the case of particular $f$ and $\zeta$ given through the data of the incident wave $u_{0}$ (cf. (1.18)), which is assumed to be at least $C^{2}$ in a $\varepsilon^{\prime}$-tubular neighborhood of the boundary $\Gamma$ with $\varepsilon^{\prime}>\varepsilon$, and if the approximate linear forms are given by (1.36), the general result of the previous theorem can be stated in a somewhat more precise form.

Corollary 2.3. Under the above general assumptions, the following error estimate holds:

$$
\left\|r_{h} \lambda_{h}-\lambda\right\|_{-1 / 2, \Gamma}+\left\|s_{h} p_{h}-p\right\|_{1 / 2, \Gamma} \leq c h^{1 / 2} \max _{|\alpha| \leq 2} \sup _{x \in \mathscr{V}_{\varepsilon}}\left|\partial^{\alpha} u_{0}(x)\right| .
$$

2.2. Consistency error estimate for the weakly singular part. The sesquilinear form $b(\cdot, \cdot)$ is defined by means of the kernel

$$
G=G_{1}+G_{2},
$$

where $G_{m}, m=1,2$, are given in (1.11). Well-known properties of the Bessel functions show that $G$ can be decomposed in the form

$$
G=E+\Phi
$$

where the kernel $E$ is given by

$$
E(x, y):=\frac{2}{\omega_{n}} F_{n}(|x-y|), \quad x \neq y,
$$

and the function $\Phi$ may be written as

$$
\Phi(x, y)= \begin{cases}u(r) & \text { if the dimension } n=3 \\ r^{2} v(r) \ln r+w(r) & \text { if } n=2\end{cases}
$$


The constant $\omega_{n}:=2 \pi^{n / 2} / \Gamma(n / 2)$ is the measure of the unit sphere of $\mathbb{R}^{n}$, the function $t \mapsto F_{n}(t)$ a suitable primitive of $t \mapsto-1 / t^{n-1}, r:=|x-y|$, and $u, v$, and $w$ are analytic functions.

Let $b^{(0)}$ and $b_{h}^{(0)}$ be the sesquilinear forms defined respectively on $\Gamma$ and $\Gamma_{h}$ through the respective kernels $\partial_{\mathbf{n}} E(\cdot, \cdot)$ and $\partial_{\mathbf{n}_{h}} E(\cdot, \cdot)$, and $b^{(1)}$ and $b_{h}^{(1)}$ the sesquilinear forms obtained in the same way via the kernel $\Phi$.

For the weakly singular part of the kernel, the consistency error estimate is established as follows.

Theorem 2.4. For all $\mu_{h}$ in $M_{h}$ and $p_{h}$ in $V_{h}$, the following estimate holds:

$$
\left|b^{(1)}\left(s_{h} p_{h}, r_{h} \mu_{h}\right)-b_{h}^{(1)}\left(p_{h}, \mu_{h}\right)\right| \leq c h^{1 / 2}\left\|p_{h}\right\|_{1 / 2}\left\|\mu_{h}\right\|_{-1 / 2} .
$$

Proof. First, we take the difference between the respective values of the kernels of the sesquilinear forms $b^{(1)}$ and $b_{h}^{(1)}$ and write it as

$$
\begin{aligned}
\partial_{\mathbf{n}} \Phi(x, y)-\partial_{\mathbf{n}_{h}} \Phi\left(x_{h}, y_{h}\right)=g(r) \frac{\mathbf{r}}{r} \cdot \mathbf{n}(y)-g\left(r_{h}\right) \frac{\mathbf{r}_{h}}{r_{h}} \cdot \mathbf{n}_{h}\left(y_{h}\right) \\
=\left(g(r)-g\left(r_{h}\right)\right) \frac{\mathbf{r}}{r} \cdot \mathbf{n}(y)+g\left(r_{h}\right) \frac{\mathbf{r}}{r} \cdot\left(\mathbf{n}(y)-\mathbf{n}_{h}\left(y_{h}\right)\right) \\
\quad+g\left(r_{h}\right)\left(\frac{\mathbf{r}}{r}-\frac{\mathbf{r}_{h}}{r_{h}}\right) \cdot \mathbf{n}_{h}\left(y_{h}\right),
\end{aligned}
$$

where $\mathbf{r}:=x-y, r:=|\mathbf{r}|, \mathbf{r}_{h}:=x_{h}-y_{h}, r_{h}:=\left|\mathbf{r}_{h}\right|$, and $g$ represents a Lipschitz function over each bounded set of $\mathbb{R}$.

Hereafter, whenever there are two variables designated by the same letter, the second being indexed by $h$, such as $x$ and $x_{h}$, it is assumed without further comment that the two points $x$ and $x_{h}$ lie respectively on $\Gamma$ and $\Gamma_{h}$ and that they are related to each other by $x=\mathscr{P} x_{h}$.

Since $x$ and $y$ are varying on $\Gamma$, one easily obtains (cf. Nédélec [25])

$$
|| x-y|-| x_{h}-y_{h}|| \leq \operatorname{ch}^{2}|x-y| \text { for all } x, y \text { on } \Gamma .
$$

It follows that

$$
\left|\left(g(r)-g\left(r_{h}\right)\right) \frac{\mathbf{r}}{r} \cdot \mathbf{n}(y)\right| \leq c h^{2}|x-y| .
$$

Therefore, the respective part of the expression for $b^{(1)}\left(s_{h} p_{h}, r_{h} \mu_{h}\right)-b_{h}^{(1)}\left(p_{h}, \mu_{h}\right)$ can be bounded by $c h^{2}\left\|p_{h}\right\|_{0}\left\|\mu_{h}\right\|_{0}$.

Using the estimates

$$
\left\{\begin{array}{l}
\left|\mathbf{n}(y)-\mathbf{n}_{h}\left(y_{h}\right)\right| \leq c h, \\
\left|\frac{\mathbf{r}}{r}-\frac{\mathbf{r}_{h}}{r_{h}}\right| \leq c h,
\end{array}\right.
$$

one can bound the remaining expressions in (2.11) to obtain terms in $c h\left\|p_{h}\right\|_{0}\left\|\mu_{h}\right\|_{0}$. The inverse inequality (1.44) leads to the estimate (2.10).

2.3. Consistency error estimate for the singular part of the kernel. Clearly, the proof of Theorem 2.1 is now reduced to that of 
Theorem 2.5. For all $\mu_{h}$ in $M_{h}$ and $p_{h}$ in $V_{h}$, the following estimate holds:

$$
\left|b^{(0)}\left(s_{h} p_{h}, r_{h} \mu_{h}\right)-b_{h}^{(0)}\left(p_{h}, \mu_{h}\right)\right| \leq c h^{1 / 2}\left\|p_{h}\right\|_{1 / 2}\left\|\mu_{h}\right\|_{-1 / 2} .
$$

Proof. The error to be estimated is denoted by

$$
e_{h}:=b^{(0)}\left(s_{h} p_{h}, r_{h} \mu_{h}\right)-b_{h}^{(0)}\left(p_{h}, \mu_{h}\right), \quad \mu_{h} \in M_{h}, p_{h} \in V_{h} .
$$

Recall that the kernel of $b^{(0)}\left(\right.$ resp. $\left.b_{h}^{(0)}\right)$ is $\partial_{\mathbf{n}} E(x, y)$ (resp. $\left.\partial_{\mathbf{n}_{h}} E\left(x_{h}, y_{h}\right)\right)$.

It is easy to see that the earlier procedure used for the weakly singular part of the kernel fails here because of the nonconvergence of certain integrals that arise.

For the sake of conciseness, throughout this section the following notation is adopted:

$$
\begin{cases}p:=s_{h} p_{h}, & p_{h} \in V_{h} \\ \mu:=r_{h} \mu_{h}, & \mu_{h} \in M_{h} .\end{cases}
$$

The error $e_{h}$ can be written as

$$
\begin{aligned}
e_{h}= & \int_{\Gamma} \int_{\Gamma} \partial_{\mathbf{n}} E(x, y) p(y) \overline{\mu(x)} d \Gamma(y) d \Gamma(x) \\
& -\int_{\Gamma_{h}} \int_{\Gamma_{h}} \partial_{\mathbf{n}_{h}} E\left(x_{h}, y_{h}\right) p_{h}\left(y_{h}\right) \overline{\mu_{h}\left(x_{h}\right)} d \Gamma_{h}\left(y_{h}\right) d \Gamma_{h}\left(x_{h}\right) .
\end{aligned}
$$

Recall that $h$ is supposed to be sufficiently small so that $\Gamma_{h}$ is contained in the $\varepsilon$-tubular neighborhood $\mathscr{V}_{\varepsilon}$. All $p_{h}$ in $V_{h}$ can be extended to $\mathscr{V}_{\varepsilon}$ in the following way:

$$
p(\tilde{y})=p_{h}\left(y_{h}\right)
$$

for all $\tilde{y}=y+\operatorname{tn}(y)$ in $\mathscr{V}_{\varepsilon}$ with $y=\mathscr{P} \tilde{y}=\mathscr{P} y_{h}$.

This extension gives a function $p$ constant along the normal $\mathbf{n}$ to $\Gamma$ and coinciding with $s_{h} p_{h}$ on $\Gamma$ and with $p_{h}$ on $\Gamma_{h}$. Note that this notation is compatible with the one given in (2.17).

In order to avoid unnecessary complications in the proofs and long discussions of every situation that may occur, in the sequel the interior domain $\Omega_{1}$ is assumed to be strictly convex. However, in other situations the proofs can be adapted to yield the same conclusions.

The remaining part of the proof will be given in a sequence of lemmas.

Lemma 2.6. Using previously introduced notations in (2.17) and (2.8), the error $e_{h}$ may be written as

$$
\begin{aligned}
& e_{h}= \frac{2}{\omega_{n}} \int_{\Gamma}\left(F_{n}(|\cdot|) * T\right)(x) \bar{\mu}(x) d \Gamma(x) \\
&+\sum_{K \in \mathscr{Y}_{h}} \int_{K}\left(\int_{\Gamma_{h}} \partial_{\mathbf{n}_{h}} E\left(x, y_{h}\right)-\int_{\Gamma_{h}} \partial_{\mathbf{n}_{h}} E\left(x_{h}, y_{h}\right)\right) \\
& \cdot\left(p\left(y_{h}\right)-p\left(x_{h}\right)\right) d \Gamma_{h}\left(y_{h}\right) \bar{\mu}_{K} d K\left(x_{h}\right),
\end{aligned}
$$


where $T$ is the compactly supported distribution of $\mathbb{R}^{n}$ given by

$$
\langle T, \Phi\rangle=\int_{\Omega_{1} \backslash \bar{\Omega}_{1}^{h}} \operatorname{grad} p \cdot \operatorname{grad} \phi d x, \quad \forall \phi \in \mathscr{D}\left(\mathbb{R}^{n}\right)
$$

and $\Omega_{1}^{h}$ the bounded domain enclosed by $\Gamma_{h}$.

Proof. Using the definition of $\left.\mu\right|_{\widetilde{K}}$, we can write the error $e_{h}$ as

$$
\begin{aligned}
e_{h}=\sum_{K \in \mathscr{S}_{h}} \int_{K}( & \int_{\Gamma} \partial_{\mathbf{n}} E(x, y) p(y) d \Gamma(y) \\
& \left.-\int_{\Gamma_{h}} \partial_{\mathbf{n}_{h}} E\left(x_{h}, y_{h}\right) p\left(y_{h}\right) d \Gamma_{h}\left(y_{h}\right)\right) \bar{\mu}_{K} d K\left(x_{h}\right) .
\end{aligned}
$$

For almost all $x$ in $\Gamma$ (i.e., such that $x_{h}$ is in the interior of any $K$ in $\mathscr{T}_{h}$ ), adding and subtracting in (2.22) the term $\int_{\Gamma_{k}} \partial_{\mathbf{n}_{h}} E\left(x, y_{h}\right) p\left(y_{h}\right) d \Gamma_{h}\left(y_{h}\right)$ leads to the following expression of $e_{h}$ :

$$
\begin{aligned}
e_{h}=\sum_{K \in \mathscr{T}_{h}} \int_{K}\left\{\int_{\Gamma} \partial_{\mathbf{n}} E(x, y) p(y) d \Gamma(y)\right. \\
\left.\quad-\int_{\Gamma_{h}} \partial_{\mathbf{n}_{k}} E\left(x, y_{h}\right) p\left(y_{h}\right) d \Gamma_{h}\left(y_{h}\right)\right\} \bar{\mu}_{K} d K\left(x_{h}\right) \\
+\sum_{K \in \mathscr{T}_{h}} \int_{K}\left\{\int_{\Gamma_{h}} \partial_{\mathbf{n}_{h}} E\left(x, y_{h}\right) p\left(y_{h}\right) d \Gamma_{h}(y)\right. \\
\left.-\int_{\Gamma_{h}} \partial_{\mathbf{n}_{h}} E\left(x_{h}, y_{h}\right) p\left(y_{h}\right) d \Gamma_{h}\left(y_{h}\right)\right\} \bar{\mu}_{K} d K\left(x_{h}\right) .
\end{aligned}
$$

In order to transform the term

$$
\begin{aligned}
& \cdot \int_{\Gamma} \partial_{\mathbf{n}} E(x, y) p(y) d \Gamma(y)-\int_{\Gamma_{h}} \partial_{\mathbf{n}_{h}} E\left(x, y_{h}\right) p\left(y_{h}\right) d \Gamma_{h}\left(y_{h}\right) \\
& \quad=\sum_{L \in \mathscr{P}_{h}}\left\{\int_{\widetilde{L}} \partial_{\mathbf{n}} E(x, y) p(y) d \widetilde{L}(y)-\int_{L} \partial_{\mathbf{n}_{h}} E\left(x, y_{h}\right) p\left(y_{h}\right) d L\left(y_{h}\right)\right\},
\end{aligned}
$$

the essential tool is to use a Green's formula applied to the functions $E(x, \cdot)$ and $p$ in the open set bounded by the exact and the approximate boundaries $\Gamma$ and $\Gamma_{h}$. In the previous formula (2.24), $\widetilde{L}=\mathscr{P} L$ is a curvilinear element on $\Gamma$ associated with $L$ in the same way as $\widetilde{K}$ was associated with $K$ in (1.38). However, such a treatment cannot be carried out directly because of the lack of regularity of the function $p$ on the latter domain in the three-dimensional case.

Consequently, the Green's formula is applied to the respective functions in each domain $U_{L}$ bounded by $L, \widetilde{L}$ and, in the three-dimensional case, in the faces $F_{L}$ generated when the normal $\mathbf{n}$ to $\Gamma$ runs over the boundary $\partial \widetilde{L}$ of $\widetilde{L}$; see Figure 1 . 


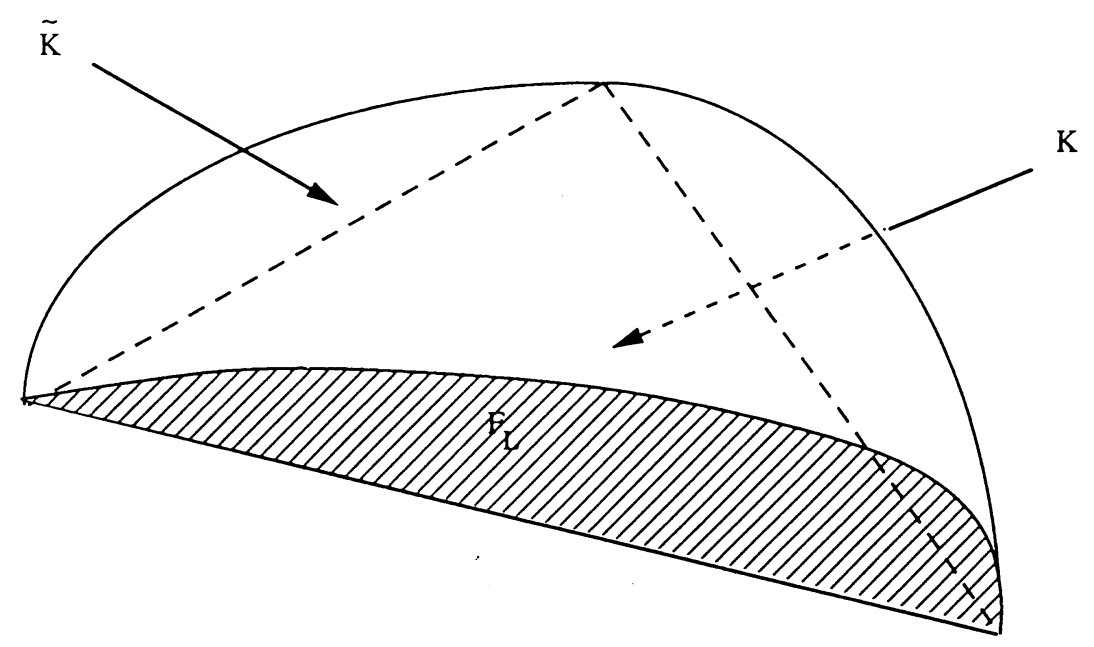

FIGURE 1

Two cases must be distinguished.

(i) The element $L$ is different from the element $K$. In this case, $x$ does not lie on $\tilde{L}$. One gets

$$
\begin{aligned}
\int_{U_{L}}(\Delta & E(x, y) p(y)-E(x, y) \Delta p(y)) d y \\
= & \int_{\widetilde{L}}\left(\partial_{\mathbf{n}} E(x, y) p(y)-E(x, y) \partial_{\mathbf{n}} p(y)\right) d \widetilde{L}(y) \\
& -\int_{L}\left(\partial_{\mathbf{n}_{h}} E\left(x, y_{h}\right) p\left(y_{h}\right)-E\left(x, y_{h}\right) \partial_{\mathbf{n}_{h}} p\left(y_{h}\right)\right) d L\left(y_{h}\right) \\
& +\int_{F_{L}}\left(\partial_{\nu} E(x, y) p(y)-E(x, y) \partial_{\nu} p(y)\right) d F_{L}(y),
\end{aligned}
$$

where $\nu$ is the unit normal to $F_{L}$ outwardly directed to $U_{L}$. Since $p$ is constant along the normal $\mathbf{n}$, the equation (2.25) can be reduced to

$$
\begin{aligned}
-\int_{U_{L}} E(x, y) \Delta p(y) d y= & \int_{\widetilde{L}} \partial_{\mathbf{n}} E(x, y) p(y) d \tilde{L}(y)+A_{L}(x) \\
& -\int_{L} \partial_{\mathrm{n}_{h}} E\left(x, y_{h}\right) p\left(y_{h}\right) d L\left(y_{h}\right) \\
& +\int_{F_{L}} \partial_{\nu} E(x, y) p(y) d F_{L}(y),
\end{aligned}
$$

where $A_{L}(x)$ is defined by

$$
A_{L}(x):=\int_{L} E\left(x, y_{h}\right) \partial_{\mathbf{n}_{h}} p\left(y_{h}\right) d L\left(y_{h}\right)-\int_{F_{L}} E(x, y) \partial_{\nu} p(y) d F_{L}(y) .
$$

(ii) The element $L$ and the element $K$ are the same element. In this case, the singularity of the function $E(x, \cdot)$ at the point $x$ forces the removal from $U_{L}$ of all points $y$ such that $|x-y|<\varepsilon$. Taking the limit as $\varepsilon$ goes to zero 
only has the effect of adding to the right-hand side of (2.26) the term

$$
\begin{array}{r}
\lim _{\varepsilon \rightarrow 0} \int_{S_{\varepsilon}} \operatorname{grad} E(x, y) \cdot \frac{x-y}{|x-y|} p(y) d S_{\varepsilon}(y) \\
=-\lim _{\varepsilon \rightarrow 0} \frac{\omega_{n}}{2} \frac{2}{\omega_{n}} F_{n}^{\prime} \varepsilon^{n-1} p\left(x_{\varepsilon}\right)=p(x),
\end{array}
$$

where $x_{\varepsilon}$ is a point in $S_{\varepsilon}:=\left\{y \in \bar{U}_{L}|| x-y \mid=\varepsilon\right\}$ whose existence follows from the mean value theorem.

Let $P$ be the function in $L^{2}\left(\mathbb{R}^{n}\right)$ defined almost everywhere by

$$
P:= \begin{cases}\Delta p(y), & y \in U_{L} \text { and } L \in \mathscr{T}_{h}, \\ 0, & \text { otherwise. }\end{cases}
$$

Summing over all $L$ in $\mathscr{T}_{h}$, we obtain

$$
\begin{aligned}
-\int_{\mathbb{R}^{n}} E(x, y) P(y) d y= & \int_{\Gamma} \partial_{\mathbf{n}} E(x, y) p(y) d \Gamma(y)+\sum_{L \in \mathscr{T}_{h}} A_{L}(x) \\
& -\int_{\Gamma_{h}} \partial_{\mathbf{n}_{h}} E\left(x, y_{h}\right) p\left(y_{h}\right) d \Gamma_{h}\left(y_{h}\right)+p(x),
\end{aligned}
$$

since

$$
\sum_{L \in \mathscr{T}_{h}} \int_{F_{L}}\left(\partial_{\nu} E(x, y) p(y)\right) d F_{L}(y)=0
$$

because $p$ is continuous across $F_{L}$ and $E(x, \cdot)$ is $C^{\infty}$ except at the point $x$.

Thus, using (2.23) and (2.30), we obtain

$$
\begin{aligned}
e_{h}= & -\int_{\Gamma} \overline{\mu(x)} \sum_{L \in \mathscr{T}_{h}} A_{L}(x) d \Gamma(x)-\int_{\Gamma} \overline{\mu(x)} p(x) d \Gamma(x) \\
& +\sum_{K \in \mathscr{T}_{h}} \int_{K} \int_{\Gamma_{h}}\left(\partial_{\mathbf{n}_{h}} E\left(x, y_{h}\right) p\left(y_{h}\right)-\partial_{\mathbf{n}_{h}} E\left(x_{h}, y_{h}\right)\right) p\left(y_{h}\right) d \Gamma_{h}\left(y_{h}\right) \bar{\mu}_{K} d K\left(x_{h}\right) \\
& -\int_{\Gamma} \overline{\mu(x)}\left(\int_{\mathbb{R}^{n}} E(x, y) P(y) d y\right) d \Gamma(x) .
\end{aligned}
$$

Using Gauss's theorem, we can write

$$
p\left(x_{h}\right)=\int_{\Gamma_{h}}\left(\partial_{\mathbf{n}_{h}} E\left(x, y_{h}\right)-\partial_{\mathbf{n}_{h}} E\left(x_{h}, y_{h}\right)\right) p\left(x_{h}\right) d \Gamma_{h}\left(y_{h}\right) .
$$

This completes the proof of the lemma, since formula (2.20) is now a straightforward consequence of the theory of distributions.

In order to bound $e_{h}$, the following lemma is proved.

Lemma 2.7. The distribution $T$ defined in (2.21) satisfies the estimate

$$
|\langle T, \phi\rangle| \leq c h|\operatorname{grad} p|_{0, \Gamma}\|\phi\|_{1, \mathbb{R}^{n}}, \quad \forall \phi \in \mathscr{D}\left(\mathbb{R}^{n}\right) .
$$

Proof. Let $L$ be in $\mathscr{T}_{h}$. Using the local orthonormal frame associated with $L$ and the parametrization of $\widetilde{L}$ given by

$$
\xi \mapsto \mathscr{P} x_{h}(\xi),
$$


we can express $U_{L}$ as

$$
U_{L}=\left\{(\xi, t) \in \mathbb{R}^{n} \mid x_{h}(\xi) \in L \text { and } f_{L}(\xi)<t<0\right\} .
$$

Here, $f_{L}$ is a $C^{\infty}$ function vanishing at all $\xi$ for which $x_{h}(\xi)$ is a vertex of $L$.

Using the coordinate system given in an open set of the $\varepsilon$-tubular neighborhood $\mathscr{V}_{\varepsilon}$ of $\Gamma$ by

$$
x(\xi, t)=y(\xi)+t \mathbf{n}(\xi)
$$

we can write

$$
\int_{U_{L}} \operatorname{grad} p \cdot \operatorname{grad} \phi d U_{L}=\int_{L} \int_{f_{L}(\xi)}^{0} \sqrt{g_{L}(\xi, t)} g_{L}^{\alpha \beta}(\xi, t) \partial_{\xi^{\alpha}} p \partial_{\xi^{\beta}} \phi d \xi d t
$$

Greek indices run from 1 to $n-1$. The contravariant components $g_{L}^{\alpha \beta}(\xi, t)$ of the metric tensor in the above integral are those related to the coordinate system $(\xi, t)$ for which the Lebesgue measure $d U_{L}$ is expressed by $d U_{L}=$ $\sqrt{g_{L}(\xi, t)} d \xi d t$. Observe that in (2.37) we use the fact that

$$
g_{L}^{\alpha n}(\xi, t)=g_{\alpha n}^{L}(\xi, t)=0, \quad \partial_{t} p=0 .
$$

We adopt here the usual convention of tensorial calculus to sum over repeated upper and lower indices.

Usual estimates in the finite element method give $\left|f_{L}(\xi)\right| \leq c h^{2}$. Since $p$ is not depending on $t$, one has

$$
\left(\int_{L} \int_{f_{L}(\xi)}^{0}\left|\partial_{\gamma} p\right|^{2} d \xi d t\right)^{1 / 2} \leq \operatorname{ch}\left(\int_{L}\left|\partial_{\gamma} p\right|^{2} d \xi\right)^{1 / 2} .
$$

The discrete Cauchy-Schwarz inequality completes the proof.

Let us decompose the error $e_{h}$ as $e_{h}=e_{h}^{1}+e_{h}^{2}$, where $e_{h}^{1}$ is the first term in the right-hand side of (2.20). For $e_{h}^{1}$, we have

Lemma 2.8. In the above notation,

$$
\frac{2}{\omega_{n}}\left|\int_{\Gamma}\left(F_{n}(|\cdot|) * T\right)(x) \bar{\mu}(x) d \Gamma(x)\right| \leq c h^{1 / 2}\left\|p_{h}\right\|_{1 / 2}\left\|\mu_{h}\right\|_{-1 / 2}
$$

for all $\mu_{h}$ in $M_{h}$ and $p_{h}$ in $V_{h}$.

Proof. Since $T$ is a compactly supported distribution in $H^{-1}\left(\mathbb{R}^{n}\right)$ and the convolution operator $F_{n}(|\cdot|) *$ is a pseudodifferential operator of order -2 , the following result holds:

$$
F_{n}(|\cdot|) * T \in H_{\mathrm{loc}}^{1}\left(\mathbb{R}^{n}\right) .
$$

Trace theorems in Sobolev spaces give

$$
\begin{aligned}
\left|\int_{\Gamma} \bar{\mu}(x)\left(F_{n}(|\cdot|) * T\right)(x) d \Gamma(x)\right| & \leq c\left\|F_{n}(|\cdot|) * T\right\|_{1 / 2, \Gamma}\left\|\mu_{h}\right\|_{-1 / 2} \\
& \leq c h\|\operatorname{grad} p\|_{0, \Gamma}\left\|\mu_{h}\right\|_{-1 / 2} .
\end{aligned}
$$

By the inverse inequalities of Lemma 1.5, one gets (2.40), proving the lemma. 
It remains to estimate the term $e_{h}^{2}$. We decompose it into three terms,

$$
e_{h}^{2}=e_{h}^{2,1}+e_{h}^{2,2}+e_{h}^{2,3}
$$

where we have set

$$
\begin{aligned}
e_{h}^{2,1}:=\sum_{K \in \mathscr{T}_{h}} \sum_{L \in \mathscr{N}_{K}} \int_{K} \int_{L}\left(\partial_{\mathbf{n}_{h}} E\left(x, y_{h}\right)-\partial_{\mathbf{n}_{h}} E\left(x_{h}, y_{h}\right)\right) \\
\cdot\left(p\left(y_{h}\right)-p\left(x_{h}\right)\right) \bar{\mu}_{K} d L\left(y_{h}\right) d K\left(x_{h}\right), \\
e_{h}^{2,2}:=\sum_{K \in \mathscr{T}_{h}} \int_{K} \int_{K}\left(\partial_{\mathbf{n}_{h}} E\left(x, y_{h}\right)-\partial_{\mathbf{n}_{h}} E\left(x_{h}, y_{h}\right)\right) \\
\cdot\left(p\left(y_{h}\right)-p\left(x_{h}\right)\right) \bar{\mu}_{K} d K\left(y_{h}\right) d K\left(x_{h}\right), \\
e_{h}^{2,3}:=\sum_{K \in \mathscr{T}_{h}} \sum_{L \in \mathscr{I}_{K}} \int_{K} \int_{L}\left(\partial_{\mathbf{n}_{h}} E\left(x, y_{h}\right)-\partial_{\mathbf{n}_{h}} E\left(x_{h}, y_{h}\right)\right) \\
\cdot\left(p\left(y_{h}\right)-p\left(x_{h}\right)\right) \bar{\mu}_{K} d L\left(y_{h}\right) d K\left(x_{h}\right),
\end{aligned}
$$

with the notations

$$
\begin{gathered}
\mathscr{N}_{K}:=\left\{L \in \mathscr{T}_{h} \mid L \cap K=\varnothing\right\}, \\
\mathscr{A}_{K}:=\left\{L \in \mathscr{T}_{h} \mid L \cap K \neq \varnothing \text { and } L \neq K\right\} .
\end{gathered}
$$

Note that, thanks to the uniform regularity of the triangulation, as assumed in (1.29), there exists a constant $c>0$ not depending on $h, L$ and $K$ such that

$$
\inf \{|x-y| ; x \in K \text { and } y \in L\} \geq c h, \quad \forall L \in \mathscr{N}_{K} .
$$

The estimation of the term $e_{h}^{2,1}$ requires the following lemma.

Lemma 2.9. For all $K$ in $\mathscr{T}_{h}$ and all $L$ in $\mathscr{N}_{K}$, the following estimate holds:

$$
\left|\left(\partial_{\mathbf{n}_{h}} E\left(x, y_{h}\right)-\partial_{\mathbf{n}_{h}} E\left(x_{h}, y_{h}\right)\right)\right| \leq \frac{c h}{\left|x-y_{h}\right|^{n-1}}
$$

for all $\left(x_{h}, y_{h}\right)$ in $K \times L$.

Proof. Set

$$
\begin{cases}\mathbf{R}:=y_{h}-x, & R:=|\mathbf{R}| \\ \mathbf{R}_{h}:=y_{h}-x_{h}, & R_{h}:=\left|\mathbf{R}_{h}\right|\end{cases}
$$

There follows

$$
\partial_{\mathbf{n}_{h}} E\left(x, y_{h}\right)-\partial_{\mathbf{n}_{h}} E\left(x_{h}, y_{h}\right)=-\frac{2}{\omega_{n}}\left(\frac{\mathbf{R}}{R^{n}}-\frac{\mathbf{R}_{h}}{R_{h}^{n}}\right) \cdot \mathbf{n}_{L},
$$

where $\mathbf{n}_{L}$ is the unit normal to the element $L$ coinciding with $\mathbf{n}_{h}$ on $L$.

Note that one can write

$$
\frac{\mathbf{R}}{R^{n}}-\frac{\mathbf{R}_{h}}{R_{h}^{n}}=\frac{\mathbf{R}-\mathbf{R}_{h}}{R^{n}}+\mathbf{R}_{h} \frac{\left(R_{h}-R\right)\left(R_{h}^{n-1}+R_{h}^{n-2} R+\cdots+R^{n-1}\right)}{R_{h}^{n} R^{n}} .
$$

Using the bounds

$$
\left|R-R_{h}\right| \leq\left|\mathbf{R}-\mathbf{R}_{h}\right| \leq c h^{2}
$$


and $R_{h} \geq c h$, a consequence of (2.49), we conclude

$$
\left|\frac{R}{R_{h}}-1\right| \leq c h
$$

which yields

$$
\left\{\begin{array}{l}
R_{h} \geq c R \\
R \geq c R_{h}
\end{array}\right.
$$

with a constant $c>0$ not depending on $h, L$ or $K$.

As a result, the estimate (2.50) follows in a straightforward way from (2.53).

Now we come to

Lemma 2.10. There exists a constant $c$ independent of $h$ such that

$$
\left|e_{h}^{2,1}\right| \leq c h^{1 / 2}\left\|p_{h}\right\|_{1 / 2}\left\|\mu_{h}\right\|_{-1 / 2}
$$

for all $\mu_{h}$ in $M_{h}$ and $p_{h}$ in $V_{h}$.

Proof. Lemma 2.9 gives

$$
\left|e_{h}^{2,1}\right| \leq c h \sum_{K \in \mathscr{T}_{h}} \sum_{L \in \mathcal{J}_{K}} \int_{K} \int_{L} \frac{\left|p\left(y_{h}\right)-p\left(x_{h}\right)\right|}{\left|y_{h}-x\right|^{n-1}}\left|\mu_{K}\right| d L\left(y_{h}\right) d K\left(x_{h}\right) .
$$

Repetition of the arguments used to prove (2.56) yields $\left|y_{h}-x\right| \geq c|y-x|$. Since $p$ is constant along the normal $\mathbf{n}$ to $\Gamma$ (i.e., $p(y)=p\left(y_{h}\right)$ if $\left.y=\mathscr{P} y_{h}\right)$, there follows

$$
\begin{aligned}
\left|e_{h}^{2,1}\right| & \leq c h \int_{\Gamma} \int_{\Gamma} \frac{|p(y)-p(x)|}{|y-x|^{n-1}}|\mu(x)| d \Gamma(y) d \Gamma(x) \\
& =\operatorname{ch} \int_{\Gamma} \int_{\Gamma} \frac{|p(y)-p(x)|}{|y-x|^{n-1-\alpha / 2}} \frac{|\mu(x)|}{|y-x|^{\alpha / 2}} d \Gamma(y) d \Gamma(x) .
\end{aligned}
$$

If $\alpha$ is chosen so that $n-3<\alpha<n-1$, the Cauchy-Schwarz inequality gives

$$
\begin{aligned}
\left|e_{h}^{2,1}\right| \leq & \operatorname{ch}\left(\int_{\Gamma} \int_{\Gamma} \frac{|p(y)-p(x)|^{2}}{|y-x|^{2(n-1)-\alpha}} d \Gamma(y) d \Gamma(x)\right)^{1 / 2} \\
& \cdot\left(\int_{\Gamma} \int_{\Gamma} \frac{|\mu(x)|^{2}}{|y-x|^{\alpha}} d \Gamma(y) d \Gamma(x)\right)^{1 / 2}
\end{aligned}
$$

Note that

$$
\left(\int_{\Gamma} \int_{\Gamma} \frac{|\mu(x)|^{2}}{|y-x|^{\alpha}} d \Gamma(y) d \Gamma(x)\right)^{1 / 2} \leq c_{\alpha}\|\mu\|_{0, \Gamma}
$$

with

$$
c_{\alpha}:=\left(\sup _{x \in \Gamma} \int_{\Gamma} \frac{d \Gamma(y)}{|x-y|^{\alpha}}\right)^{1 / 2} .
$$

Setting $n-1-\alpha=2 s$, if $0<s<1$ (i.e., $\alpha>n-3$ ), one gets the bound (cf. Lions and Magenes [23])

$$
\left(\int_{\Gamma} \int_{\Gamma} \frac{|p(y)-p(x)|^{2}}{|y-x|^{(n-1)+2 s}} d \Gamma(y) d \Gamma(x)\right)^{1 / 2} \leq\|p\|_{s, \Gamma}
$$


which yields

$$
\left|e_{h}^{2,1}\right| \leq c_{s} h\|p\|_{s, \Gamma}\|\mu\|_{0, \Gamma} \quad \text { for all } s \text { with } 0<s<1 .
$$

Using the inverse inequality, one can write

$$
\left|e_{h}^{2,1}\right| \leq c_{s} h^{1 / 2}\|p\|_{s, \Gamma}\|\mu\|_{-1 / 2, \Gamma} \text { for all } s \text { with } 0<s<1 .
$$

It suffices to take $s=1 / 2$, thus $\alpha=n-2$, to complete the proof of the lemma.

Remark 2.11. In the bidimensional case (i.e., $n=2$ ), we have $\alpha=0$. This makes it possible to obtain directly the estimate (2.58) by means of the CauchySchwarz inequality (cf. Bendali [2]). This is no longer possible for threedimensional problems.

The next lemma gives an estimate for the term $e_{h}^{2,2}$.

Lemma 2.12. There exists a constant $c$ independent of $h$ such that

$$
\left|e_{h}^{2,2}\right| \leq \operatorname{ch}\left\|p_{h}\right\|_{1 / 2}\left\|\mu_{h}\right\|_{-1 / 2}
$$

for all $\mu_{h}$ in $M_{h}$ and $p_{h}$ in $V_{h}$.

Proof. Since $p_{K}$ is in $\mathbb{P}_{1}$, the estimate of the term $e_{h}^{2,2}$ is obtained as follows:

$$
\begin{aligned}
e_{h}^{2,2}= & \sum_{K \in \mathscr{T}_{h}} \int_{K} \int_{K} \partial_{\mathbf{n}_{K}} E\left(x, y_{h}\right) \operatorname{grad}_{K} p_{K} \cdot \mathbf{R}_{h} \bar{\mu}_{K} d K\left(y_{h}\right) d K\left(x_{h}\right) \\
= & \sum_{K \in \mathscr{T}_{h}} \int_{K} \int_{K} \partial_{\mathbf{n}_{K}} E\left(x, y_{h}\right) \operatorname{grad}_{K} p_{K} \cdot \mathbf{R} \bar{\mu}_{K} d K\left(y_{h}\right) d K\left(x_{h}\right) \\
& +\sum_{K \in \mathscr{T}_{h}} \int_{K} \int_{K} \partial_{\mathbf{n}_{K}} E\left(x, y_{h}\right) \operatorname{grad}_{K} p_{K} \cdot\left(\mathbf{R}_{h}-\mathbf{R}\right) \bar{\mu}_{K} d K\left(y_{h}\right) d K\left(x_{h}\right) .
\end{aligned}
$$

Here, $\mathbf{R}$ and $\mathbf{R}_{h}$ are defined as in (2.51), and $\mathbf{n}_{K}$ in the same way as $\mathbf{n}_{L}$.

Since $\operatorname{grad}_{K} p_{K} \cdot\left(\mathbf{R}_{h}-\mathbf{R}\right)$ is constant as a function of $y_{h}$ on $K$, there follows (2.67)

$$
\begin{aligned}
& \sum_{K \in \mathscr{T}_{h}} \int_{K} \int_{K} \partial_{\mathbf{n}_{K}} E\left(x, y_{h}\right) \operatorname{grad}_{K} p_{K} \cdot\left(\mathbf{R}_{h}-\mathbf{R}\right) \bar{\mu}_{K} d K\left(y_{h}\right) d K\left(x_{h}\right) \\
& \quad=\sum_{K \in \mathscr{F}_{h}} \int_{K} \bar{\mu}_{K} \operatorname{grad}_{K} p_{K} \cdot\left(\mathbf{R}_{h}-\mathbf{R}\right)\left(\int_{K} \partial_{\mathbf{n}_{K}} E\left(x, y_{h}\right) d K\left(y_{h}\right)\right) d K\left(x_{h}\right) .
\end{aligned}
$$

The inner integral can be calculated by Gauss's theorem:

$$
\int_{K} \partial_{\mathbf{n}_{K}} E\left(x, y_{h}\right) d K\left(y_{h}\right)=2 \frac{\omega(K, x)}{\omega_{n}},
$$

where $\omega(K, x)$ is the angle (resp. the solid angle) under which the element $K$ is viewed from the point $x$ for $n=2$ (resp. $n=3$ ).

Using (2.54), we can bound the term (2.67) by

$$
c h^{2} \sum_{K \in \mathscr{T}_{h}} \int_{K}\left|\operatorname{grad}_{K} p_{K}\right|\left|\mu_{K}\right| d K\left(x_{h}\right) .
$$


The Cauchy-Schwarz inequality and the inverse property of Lemma 1.5 yield

$$
\begin{aligned}
& \left|\sum_{K \in \mathscr{T}_{h}} \int_{K} \int_{K} \partial_{\mathbf{n}_{K}} E\left(x, y_{h}\right) \operatorname{grad}_{K} p_{K} \cdot\left(\mathbf{R}_{h}-\mathbf{R}\right) \bar{\mu}_{K} d K\left(y_{h}\right) d K\left(x_{h}\right)\right| \\
& \quad \leq \operatorname{ch}\left\|p_{h}\right\|_{1 / 2}\left\|\mu_{h}\right\|_{-1 / 2} .
\end{aligned}
$$

The last term to estimate in $e_{h}^{2,2}$ becomes

$$
\tilde{e}_{h}^{2,2}:=\sum_{K \in \mathscr{T}_{h}} \int_{K} \int_{K} \partial_{\mathbf{n}_{K}} E\left(x, y_{h}\right) \operatorname{grad}_{K} p_{K} \cdot \mathbf{R} \bar{\mu}_{K} d K\left(y_{h}\right) d K\left(x_{h}\right) .
$$

A straightforward calculation gives

$$
\begin{aligned}
\partial_{\mathbf{n}_{K}} E\left(x, y_{h}\right) \operatorname{grad}_{K} p_{K} \cdot \mathbf{R} & =-\frac{2}{\omega_{n}} \frac{\mathbf{R} \cdot \mathbf{n}_{K}}{R_{h}^{n}} \operatorname{grad}_{K} p_{K} \cdot \mathbf{R} \\
& =\mathbf{R} \cdot \mathbf{n}_{K} \operatorname{grad}_{K} p_{K} \cdot \operatorname{grad}_{K} E\left(x, y_{h}\right) .
\end{aligned}
$$

Since $\mathbf{R} \cdot \mathbf{n}_{K}$ is constant when $x$ is fixed and $y_{h}$ runs over $K$, the term $\tilde{e}_{h}^{2,2}$ may be written as

$$
\tilde{e}_{h}^{2,2}=\sum_{K \in \mathscr{T}_{h}} \int_{K} \bar{\mu}_{K} \mathbf{R} \cdot \mathbf{n}_{K} \operatorname{grad}_{K} p_{K} \cdot\left(\int_{K} \operatorname{grad}_{K} E\left(x, y_{h}\right) d K\left(y_{h}\right)\right) d K\left(x_{h}\right) .
$$

Two cases need to be distinguished.

(i) Two-dimensional case $(n=2)$. In this case, $K$ is a segment having $A_{K}$ and $B_{K}$ as endpoints. If $\tau_{K}$ denotes the unit tangent vector oriented from $A_{K}$ toward $B_{K}$, an elementary theorem of integral calculus gives

$$
\begin{aligned}
\int_{K} \operatorname{grad}_{K} E\left(x, y_{h}\right) d K\left(y_{h}\right) & =\left(E\left(x, B_{K}\right)-E\left(x, A_{K}\right)\right) \tau_{K} \\
& =-\frac{1}{\pi}\left(\ln \left(\left|x-B_{K}\right|\right)-\ln \left(\left|x-A_{K}\right|\right)\right) \tau_{K} .
\end{aligned}
$$

Since $\left|\mathbf{R} \cdot \mathbf{n}_{K}\right|$ is the distance of the point $x$ to the segment $K$, we have the bound

$$
\left|\tilde{e}_{h}^{2,2}\right| \leq \frac{2}{\pi} \sum_{K \in \mathscr{T}_{h}} \int_{K}\left|\mu_{K}\right|\left|\operatorname{grad}_{K} p_{K}\right|\left|\mathbf{R} \cdot \mathbf{n}_{K} \ln \left(\left|\mathbf{R} \cdot \mathbf{n}_{K}\right|\right)\right| d K\left(x_{h}\right) .
$$

Since $\left|\mathbf{R} \cdot \mathbf{n}_{K}\right| \leq c h^{2}$ and $\left|\mathbf{R} \cdot \mathbf{n}_{K}\right|^{\varepsilon}\left|\ln \left(\left|\mathbf{R} \cdot \mathbf{n}_{K}\right|\right)\right|$ is bounded for any fixed $\varepsilon>0$, it follows that for all $\varepsilon$ such that $0<\varepsilon<1 / 2$ there exists $h_{\varepsilon}^{*}>0$ and a constant $c_{\varepsilon}$ not depending on $0<h<h_{\varepsilon}^{*}$ such that

$$
\left|\tilde{e}_{h}^{2,2}\right| \leq c_{\varepsilon} h^{1-\varepsilon}\left\|p_{h}\right\|_{1 / 2}\left\|\mu_{h}\right\|_{-1 / 2} \text {. }
$$

(ii) Three-dimensional case $(n=3)$. Following the method initiated by De Hoop (cf. Van Herk [36]), we can express

$$
\int_{K} \operatorname{grad}_{y_{h}} \frac{1}{\left|x-y_{h}\right|} d K\left(y_{h}\right)=\mathbf{n}_{K} \omega(K, x)+\sum_{m=1}^{3} \nu_{m}^{K} \ln \frac{R_{m+1}^{K}+\mathbf{R}_{m+1}^{K} \cdot \boldsymbol{\tau}_{m}^{K}}{R_{m}^{K}+\mathbf{R}_{m}^{K} \cdot \boldsymbol{\tau}_{m}^{K}}
$$

where $A_{1}, A_{2}$, and $A_{3}$ are the three vertices of the triangle $K, \mathbf{R}_{m}^{K}:=A_{m}-x$, $R_{m}^{K}:=\left|\mathbf{R}_{m}^{K}\right|, \boldsymbol{\tau}_{m}^{K}:=\left(A_{m+1}-A_{m}\right) /\left|A_{m+1}-A_{m}\right|$, for $m=1,2,3$; see Figure 2 . The index convention that $m+1=1$ when $m=3$ is adopted. 


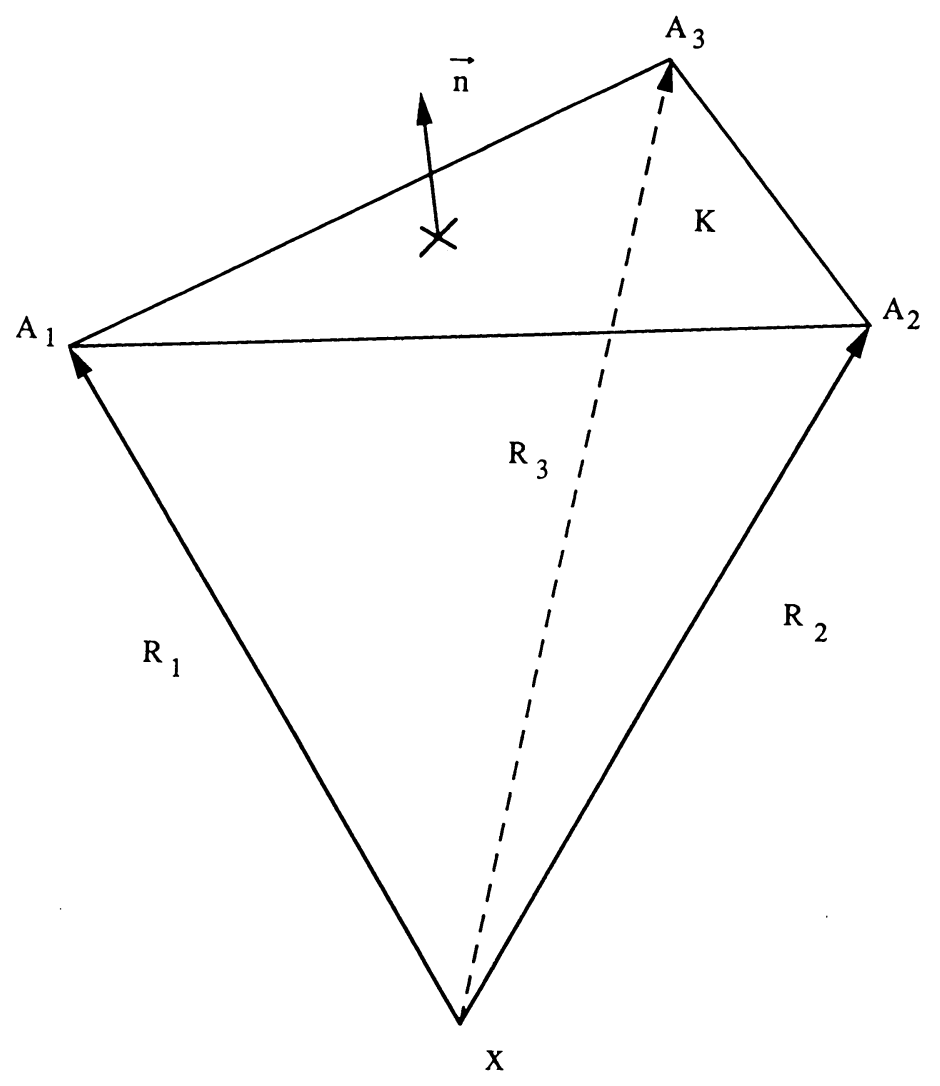

FIGURE 2

An elementary calculation gives

$$
\ln \frac{R_{m+1}^{K}+\mathbf{R}_{m+1}^{K} \cdot \tau_{m}^{K}}{R_{m}^{K}+\mathbf{R}_{m}^{K} \cdot \tau_{m}^{K}}=-\ln \left(R_{m+1}^{K}-\mathbf{R}_{m+1}^{K} \cdot \boldsymbol{\tau}_{m}^{K}\right)+\ln \left(R_{m}^{K}-\mathbf{R}_{m}^{K} \cdot \boldsymbol{\tau}_{m}^{K}\right),
$$

thus yielding

$$
\forall \varepsilon>0, \exists c_{\varepsilon}:\left|\mathbf{n}_{K} \cdot \mathbf{R}\right|^{\varepsilon}\left|\ln \frac{R_{m+1}^{K}+\mathbf{R}_{m+1}^{K} \cdot \boldsymbol{\tau}_{m}^{K}}{R_{m}^{K}+\mathbf{R}_{m}^{K} \cdot \tau_{m}^{K}}\right| \leq c_{\varepsilon} .
$$

This completes the proof of the lemma.

It remains to estimate the term $e_{h}^{2,3}$. For this, we need the following lemma.

Lemma 2.13. For all $K$ in $\mathscr{T}_{h}$ and $L$ in $\mathscr{A}_{K}$, and for all $x_{h}$ in the interior of $K$, we have

$$
\left\{\begin{array}{l}
\omega(L, x) \leq c h \\
\omega\left(L, x_{h}\right) \leq c h
\end{array}\right.
$$

The notation is that introduced for $K$ in (2.68).

Proof. This is a consequence of well-known facts about error estimates for the derivatives in finite element interpolation. The details of how to obtain these estimates are not given. However, note that these estimates are obvious in 
the two-dimensional case, and a consequence of the classical formula explicitly giving the expression of $\omega(L, x)$ and $\omega\left(L, x_{h}\right)$ in the three-dimensional case (cf., e.g., Van Herk [36]).

The last estimate is given by the following lemma.

Lemma 2.14. There exists a constant $c$ independent of $h$ such that

$$
\left|e_{h}^{2,3}\right| \leq c h^{1 / 2}\left\|p_{h}\right\|_{1 / 2}\left\|\mu_{h}\right\|_{-1 / 2}
$$

for all $\mu_{h}$ in $M_{h}$ and $p_{h}$ in $V_{h}$.

Proof. Consider two elements in $\mathscr{T}_{h}$ such that $L$ and $K$ are different, but have at least one common point $M$. Recall the notations $p_{K}:=\left.p_{h}\right|_{K}$ and $p_{L}:=\left.p_{h}\right|_{L}$. Arguments previously used give

$$
\begin{aligned}
p_{L}\left(y_{h}\right)-p_{K}\left(x_{h}\right) & =p_{L}\left(y_{h}\right)-p_{L}(M)+p_{K}(M)-p_{K}\left(x_{h}\right) \\
& =\left(y_{h}-M\right) \cdot \operatorname{grad}_{L} p_{L}+\left(M-x_{h}\right) \cdot \operatorname{grad}_{K} p_{K} \\
& =\mathbf{R} \cdot \operatorname{grad}_{L} p_{L}+(x-M) \cdot \operatorname{grad}_{L} p_{L}+\left(M-x_{h}\right) \cdot \operatorname{grad}_{K} p_{K} .
\end{aligned}
$$

Observing that $(x-M) \cdot \operatorname{grad}_{L} p_{L}+\left(M-x_{h}\right) \cdot \operatorname{grad}_{K} p_{K}$ is constant in $y_{h}$, we get

$$
\begin{aligned}
e_{h}^{2,3}= & \frac{2}{\omega_{n}} \sum_{K \in \mathscr{T}_{h}} \sum_{L \in \mathscr{A}_{K}} \int_{K}\left((x-M) \cdot \operatorname{grad}_{L} p_{L}+\left(M-x_{h}\right) \cdot \operatorname{grad}_{K} p_{K}\right) \\
& \cdot \frac{2}{\omega_{n}} \sum_{K \in \mathscr{T}_{h}} \sum_{L \in \mathscr{A}_{K}} \int_{K} \int_{L}\left(\frac{\mathbf{R}}{R^{n}}-\frac{\mathbf{R}_{h}}{R_{h}^{n}}\right) \cdot \mathbf{n}_{L} \mathbf{R} \cdot \operatorname{grad}_{L} p_{L} \bar{\mu}_{K} d L\left(y_{h}\right) d K\left(x_{h}\right) .
\end{aligned}
$$

Clearly,

$$
\left\{\begin{array}{l}
|x-M| \leq c h \\
\left|x_{h}-M\right| \leq c h
\end{array}\right.
$$

It follows from Lemma 2.13 that the first term of the right-hand side of (2.83) can be bounded by

$$
c h^{2} \sum_{K \in \mathscr{T}_{h}} \sum_{L \in \mathscr{A}_{K}} \int_{K}\left(\left|\operatorname{grad}_{K} p_{K}\right|+\left|\operatorname{grad}_{L} p_{L}\right|\right)\left|\mu_{K}\right| d L\left(y_{h}\right) d K\left(x_{h}\right)
$$

The uniform regularity of the triangulation $\mathscr{T}_{h}$ (cf. (1.29)) insures that there exists a positive integer $N$ not depending on $K$ or $h$ that bounds the number of elements of $\mathscr{A}_{K}$, i.e.,

$$
\operatorname{Card} \mathscr{A}_{K} \leq N
$$

Clearly, arguments used above enable us to bound the expression (2.85) by terms of the form $c h\left\|\mu_{h}\right\|_{-1 / 2}\left\|p_{h}\right\|_{1 / 2}$.

Introducing the common point $M$ in the second term of (2.83), thus writing

$$
\frac{\mathbf{R} \cdot \mathbf{n}_{L}}{R^{n}} \mathbf{R} \cdot \operatorname{grad}_{L} p_{L}=(M-x) \cdot \mathbf{n}_{L} \frac{\mathbf{R}}{R^{n}} \cdot \operatorname{grad}_{L} p_{L},
$$




$$
\begin{aligned}
& \frac{\mathbf{R}_{h} \cdot \mathbf{n}_{L}}{R_{h}^{n}} \mathbf{R} \cdot \operatorname{grad}_{L} p_{L} \\
& \quad=\left(M-x_{h}\right) \cdot \mathbf{n}_{L} \frac{\mathbf{R}_{h}}{R_{h}^{n}} \cdot \operatorname{grad}_{L} p_{L}+\frac{\mathbf{R}_{h} \cdot \mathbf{n}_{L}}{R_{h}^{n}}\left(\mathbf{R}-\mathbf{R}_{h}\right) \cdot \operatorname{grad}_{L} p_{L}
\end{aligned}
$$

helps estimating the term

$$
\begin{aligned}
\tilde{e}_{h}^{2,3}:= & \sum_{K \in \mathscr{T}_{h}} \sum_{L \in \mathscr{A}_{K}}\left\{\int_{K}(M-x) \cdot \mathbf{n}_{L} \bar{\mu}_{K} \operatorname{grad}_{L} p_{L} \cdot \mathbf{g}_{L}(x) d K\left(x_{h}\right)\right. \\
& \left.-\int_{K}\left(M-x_{h}\right) \cdot \mathbf{n}_{L} \bar{\mu}_{K} \operatorname{grad}_{L} p_{L} \cdot \mathbf{g}_{L}\left(x_{h}\right) d K\left(x_{h}\right)\right\} \\
& -\frac{2}{\omega_{n}} \sum_{K \in \mathscr{S}_{h}} \sum_{L \in \mathscr{A}_{K}} \int_{K}\left(\mathbf{R}-\mathbf{R}_{h}\right) \cdot \operatorname{grad}_{L} p_{L} \bar{\mu}_{K} \omega\left(L, x_{h}\right) d K\left(x_{h}\right) .
\end{aligned}
$$

The vector-valued function defined for $z$ in $\mathbb{R}^{n}$ by

$$
\mathbf{g}_{L}(z):=\int_{L} \operatorname{grad}_{L} E\left(z, y_{h}\right) d L\left(y_{h}\right)
$$

is introduced to make the preceding formulae more concise. Using the estimates

$$
\left\{\begin{array}{l}
\left|(M-x) \cdot \mathbf{n}_{L}\right| \leq c h^{2}, \\
\left|\left(M-x_{h}\right) \cdot \mathbf{n}_{L}\right| \leq c h^{2}, \\
\left|\mathbf{R}-\mathbf{R}_{h}\right| \leq c h^{2}
\end{array}\right.
$$

the De Hoop method (cf. (2.77)) and previously invoked arguments, we get

$$
\left|\tilde{e}_{h}^{2,3}\right| \leq c_{\varepsilon} h^{1-\varepsilon}\left\|\mu_{h}\right\|_{-1 / 2}\left\|p_{h}\right\|_{1 / 2} .
$$

Combining all the estimates given in the previous lemmas will prove Theorem 2.5, and hence the main results given in Theorem 2.1.

Remark 2.15. The convergence rate $h^{1 / 2}$ of the discrete solution to the exact one seems to be somewhat weak. This is due to the fact that some estimates are given in the norm of $H^{-1 / 2}(\Gamma)$, because this space is necessary for the coerciveness of the sesquilinear forms. However, in similar situations (cf. Bendali [2]), numerical results show that the convergence rate in the stronger norm of $L^{2}(\Gamma)$ is of the order $h$. Nevertheless, the $h^{1 / 2}$-estimate may be considered as a consistency result, which makes the numerical analysis of the exact problem possible: it establishes existence, uniqueness, stability of the solution of the discrete problem, and its convergence to the solution of the continuous problem. The technical assumptions on the triangulation (cf. (1.28), (1.29), and (1.30)) seem to be needed only for purposes of theoretical asymptotic analysis and are not essential in practical computations.

\section{ACKNOWLEDGMENT}

The authors wish to thank the referee for his thorough reading and useful remarks. 


\section{BIBLIOGRAPHY}

1. S. Agmon, A. Douglis, and L. Nirenberg, Estimates near the boundary for solutions of elliptic partial differential equations satisfying general boundary conditions. Part II, Comm. Pure Appl. Math. 27 (1964), 35-92.

2. A. Bendali, Approximation par éléments finis de surface de problèmes de diffraction d'ondes électromagnétiques, Thèse de Doctorat d'Etat Es-Sciences Mathématiques, Univ. P. \& M. Curie, Paris VI, Paris, 1984.

3. _ Numerical analysis of the exterior boundary value problem for the time-harmonic Maxwell equations by a boundary finite element method. Part 2. The discrete problem, Math. Comp. 43 (1984), 47-68.

4. Y. Chang and R. F. Harrington, A surface formulation for characteristic modes of material bodies, Technical Report no. 2 AD/A 000285 NTIS U.S. Department of Commerce, 1974.

5. J. Chazarain and A. Piriou, Introduction to the theory of linear partial differential equations, North-Holland, Amsterdam and New York, i 982.

6. D. Clair, Caractérisation du rayonnement plan $E$ de cornets sectoraux plan $H$ à l'aide d'une méthode bidimensionnelle, Thèse de Doctorat de $3^{\text {ème }}$ cycle, Univ. P. \& M. Curie, Paris VI, Paris, 1983.

7. D. Colton and R. Kress, Integral equation methods in scattering theory, Wiley, New York, 1983.

8. M. Costabel, Boundary integral operators on Lipschitz domains: Elementary results, SIAM J. Math. Anal. 19 (1988), 613-626.

9. M. Costabel and E. Stephan, A direct boundary integral equation method for transmission problems, J. Math. Anal. Appl. 372 (1985), 367-413.

10. M. Costabel and W. L. Wendland, Strong ellipticity of boundary integral operators, J. Reine Angew. Math. 372 (1986), 39-63.

11. N. Germain, Cours de mécanique des milieux continus, Masson, Paris, 1973.

12. J. Giroire, Integral equation methods for exterior problem for Helmholtz equation, Rapport interne no. 40, Centre de Mathématiques Appliquées, Ecole Polytechnique, Palaiseau, France, 1978.

13. __ Integral equation methods for the Helmholtz equation, Integral Equations Operator Theory 5 (1982), 505-517.

14. N. M. Gunter, Potential theory and its application to basic problems of mathematical physics, Ungar, New York, 1967.

15. M. A. Hamdi, Une formulation variationnelle par équations intégrales pour la résolution de l'équation de Helmholtz avec des conditions aux limites mixtes, C. R. Acad. Sci. Paris Sér. II Méc. Phys. Chim. Sci. Univers Sci. Terre 292 (1981), 17-20.

16. R. F. Harrington, Characteristic modes for antennas and scatterers, Numerical and Asymptotic Techniques in Electromagnetics (R. Mittra, ed.), Topics in Applied Physics, vol. 3, Springer-Verlag, Berlin, Heidelberg, and New York, 1975, pp. 51-87.

17. G. C. Hsiao and W. L. Wendland, A finite element method for some integral equations of the first kind, J. Math. Anal. Appl. 58 (1977), 449-481.

18. C. Johnson and J. C. Nédélec, On the coupling of the boundary integral and finite element methods, Math. Comp. 35 (1980), 1063-1079.

19. R. E. Kleinman and P. A. Martin, On single integral equations for the transmission problem of acoustics, SIAM J. Appl. Math. 48 (1988), 307-325.

20. R. Kress and G. F. Roach, Transmission problems for the Helmholtz equation, J. Math. Phys. 19 (1978), 1433-1437.

21. G. Krishnasamy, F. Rizzo, and T. Rudolphi, Hypersingular boundary integral equations: Their occurrence, interpretation, regularization, and computation, Developments in Boundary Elements Methods, vol. 7: Adv. Dynamic Analysis (P. K. Banerjee and S. Kobayashi, eds.), Elsevier, Amsterdam, 1991, pp. 207-252.

22. M. N. Le Roux. Méthode d'éléments finis pour la résolution numérique de problèmes extérieurs en dimension 2, RAIRO Anal. Numér. 11 (1977), 27-60. 
23. J. L. Lions and E. Magenes, Problèmes aux limites non homogènes et applications, Vol. 1, Dunod, Paris, 1968.

24. J. C. Nédélec, Approximations des équations intégrales en mécanique et en physique, Cours EDF-CEA-IRIA, Centre de Mathématiques Appliquées, Ecole Polytechnique, Palaiseau, France, 1977.

25. _ Curved finite element methods for the solution of singular integral equations on surfaces in $\mathbb{R}^{3}$, Comput. Methods Appl. Mech. Engrg. 8 (1976), 61-80.

26. Integral equations with non-integrable kernels, Integral Equations Operator Theory 5 (1982), 561-572.

27. J. C. Nédélec and J. Planchard, Une méthode variationnelle d'éléments finis pour la résolution d'un problème extérieur dans $\mathbb{R}^{3}$, RAIRO Sér. Rouge 7 (1973), 105-129.

28. U. Neri, Singular integrals, Lecture Notes in Math., vol. 200, Springer-Verlag, Berlin, Heidelberg, and New York, 1971.

29. R. S. Phillips, On the exterior problem for the reduced wave equation, Proc. Sympos. Pure Math., vol. 23, Partial Differential Equations (D. C. Spencer, ed.), Amer. Math. Soc., Providence, RI, 1973, pp. 153-160.

30. F. Rellich, Über das asymptotische Verhalten der Lösungen von $\Delta u+\lambda u=0$ in unendlichen Gebieten, Jahresber. Deutsch. Math.-Verein. 53 (1943), 57-65.

31. V. Rokhlin, Solution of acoustic scattering problems by means of second kind integral equations, Wave Motion 5 (1983), 257-272.

32. G. Schwab and W. L. Wendland, On numerical cubatures of singular surface integrals in boundary element methods, Numer. Math. 62 (1992), 343-369.

33. R. Seeley, Topics in pseudo-differential operators, Pseudo-Differential Operators (L. Nirenberg, ed.), CIME, Stresa II ciclo, Aug. 26-Sept. 3, 1968, Edizioni Cremonese, Roma, 1969.

34. A. Sequeira, The coupling of boundary integral and finite element methods for the bidimensional steady Stokes problem, Rapport interne no. 82, Centre de Mathématiques Appliquées, Ecole Polytechnique, Palaiseau, France, 1982.

35. G. Strang and G. J. Fix, An analysis of the finite-element method, Prentice-Hall, Englewood Cliffs, NJ, 1973.

36. A. Van Herk, Three dimensional analysis of magnetic fields in recording head configuration, IEEE Trans. Magnetics 5 MAG-16 (1980), 890-892.

37. C. H. Wilcox, Scattering theory for the D'Alembert equation in exterior domains, Lecture Notes in Math., vol. 442, Springer-Verlag, Berlin, Heidelberg, New York, 1975.

Laboratoire de Mathématiques Appliquées, Université de Pau \& U.R.A. CNRS 1204, I.P.R.A., Ave DE L'Université, 64000 PaU, France

E-mail address: bendali@iprvs1.univ-pau.fr

Institut de Mathematiques, USTHB, BP 32 El-Alia Bab-Ezzouar, 16111 Algiers, ALgeria 